\title{
Two modes of surface roughening during plasma etching of silicon: Role of ionized etch products
}

\section{AUTHOR(S):}

Nakazaki, Nobuya; Tsuda, Hirotaka; Takao, Yoshinori; Eriguchi, Koji; Ono, Kouichi

\section{CITATION:}

Nakazaki, Nobuya ...[et al]. Two modes of surface roughening during plasma etching of silicon: Role of ionized etch products. Journal of Applied Physics 2014, 116(22): 223302.

\section{ISSUE DATE:}

2014-12-14

URL:

http://hdl.handle.net/2433/193256

\section{RIGHT:}

Copyright 2014 American Institute of Physics. This article may be downloaded for personal use only. Any other use requires prior permission of the author and the American Institute of Physics 


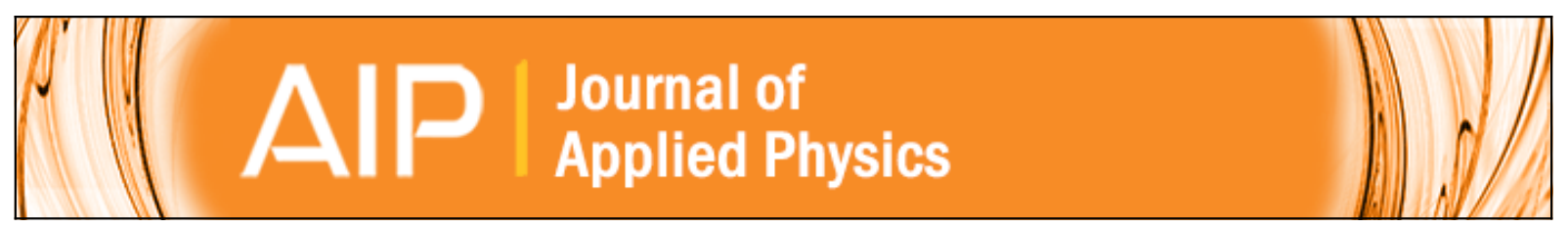

Two modes of surface roughening during plasma etching of silicon: Role of ionized etch products

Nobuya Nakazaki, Hirotaka Tsuda, Yoshinori Takao, Koji Eriguchi, and Kouichi Ono

Citation: Journal of Applied Physics 116, 223302 (2014); doi: 10.1063/1.4903956

View online: http://dx.doi.org/10.1063/1.4903956

View Table of Contents: http://scitation.aip.org/content/aip/journal/jap/116/22?ver=pdfcov

Published by the AIP Publishing

\section{Articles you may be interested in}

Surface roughening and rippling during plasma etching of silicon: Numerical investigations and a comparison with experiments

J. Vac. Sci. Technol. B 32, 031212 (2014); 10.1116/1.4874309

Modeling of implantation and mixing damage during etching of $\mathrm{SiO} 2$ over $\mathrm{Si}$ in fluorocarbon plasmas

J. Vac. Sci. Technol. A 29, 051306 (2011); 10.1116/1.3626533

Surface roughness generated by plasma etching processes of silicon

J. Vac. Sci. Technol. B 26, 1281 (2008); 10.1116/1.2932091

Surface roughening of silicon, thermal silicon dioxide, and low- $\mathrm{k}$ dielectric coral films in argon plasma

J. Vac. Sci. Technol. A 26, 151 (2008); 10.1116/1.2821747

Smoothening mechanism for GaAs(100) surfaces during ion-enhanced plasma etching

Appl. Phys. Lett. 88, 161916 (2006); 10.1063/1.2196063

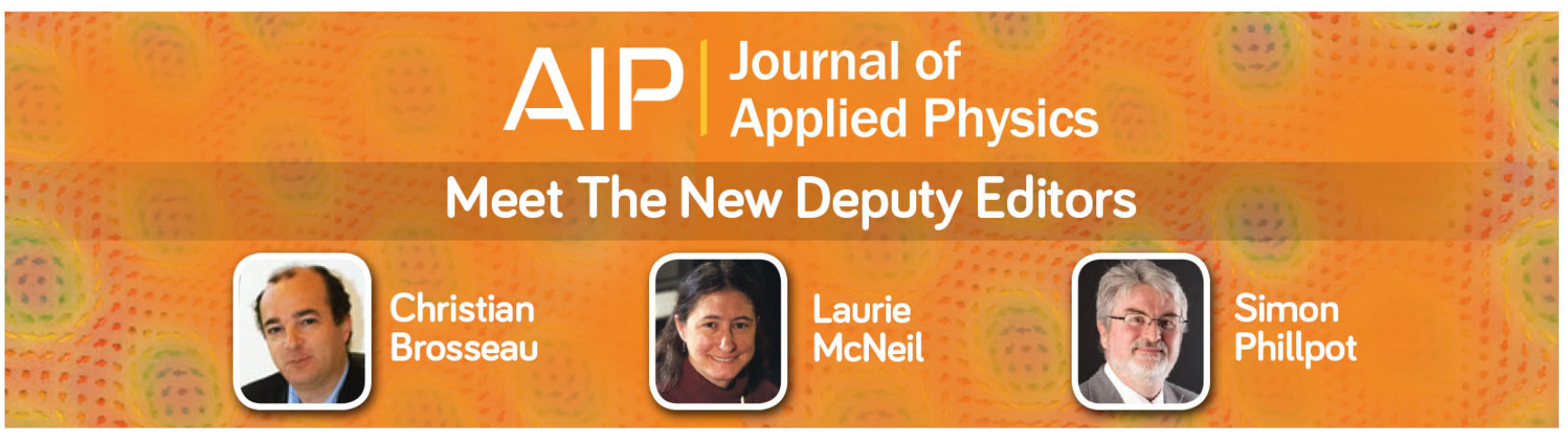




\title{
Two modes of surface roughening during plasma etching of silicon: Role of ionized etch products
}

\author{
Nobuya Nakazaki, ${ }^{\text {a) }}$ Hirotaka Tsuda, ${ }^{\text {b) }}$ Yoshinori Takao, ${ }^{\text {c) }}$ Koji Eriguchi, and Kouichi Ono d) \\ Department of Aeronautics and Astronautics, Graduate School of Engineering, Kyoto University, \\ Kyoto-daigaku Katsura, Nishikyo-ku, Kyoto 615-8540, Japan
}

(Received 6 September 2014; accepted 28 November 2014; published online 12 December 2014)

\begin{abstract}
Atomic- or nanometer-scale surface roughening has been investigated during Si etching in inductively coupled $\mathrm{Cl}_{2}$ plasmas, as a function of rf bias power or ion incident energy $E_{i}$, by varying feed gas flow rate, wafer stage temperature, and etching time. The experiments revealed two modes of surface roughening which occur depending on $E_{i}$ : one is the roughening mode at low $E_{i}<200-300$ $\mathrm{eV}$, where the root-mean-square (rms) roughness of etched surfaces increases with increasing $E_{i}$, exhibiting an almost linear increase with time during etching $(t<20 \mathrm{~min})$. The other is the smoothing mode at higher $E_{i}$, where the rms surface roughness decreases substantially with $E_{i}$ down to a low level $<0.4 \mathrm{~nm}$, exhibiting a quasi-steady state after some increase at the initial stage $(t<1 \mathrm{~min})$. Correspondingly, two different behaviors depending on $E_{i}$ were also observed in the etch rate versus $\sqrt{E_{i}}$ curve, and in the evolution of the power spectral density distribution of surfaces. Such changes from the roughening to smoothing modes with increasing $E_{i}$ were found to correspond to changes in the predominant ion flux from feed gas ions $\mathrm{Cl}_{x}{ }^{+}$to ionized etch products $\mathrm{SiCl}_{x}{ }^{+}$caused by the increased etch rates at increased $E_{i}$, in view of the results of several plasma diagnostics. Possible mechanisms for the formation and evolution of surface roughness during plasma etching are discussed with the help of Monte Carlo simulations of the surface feature evolution and classical molecular dynamics simulations of etch fundamentals, including stochastic roughening and effects of ion reflection and etch inhibitors. ( 2014 AIP Publishing LLC.
\end{abstract}

[http://dx.doi.org/10.1063/1.4903956]

\section{INTRODUCTION}

Plasma etching is now widely employed for manufacturing integrated devices, such as ultra-large-scale integration (ULSI) devices ${ }^{1,2}$ and microelectromehanical systems (MEMS). ${ }^{3}$ As the device dimensions continue to be scaled down, ${ }^{4}$ increasingly strict requirements are being imposed on plasma etching technology, ${ }^{5}$ including the precise control of profile, critical dimension (CD), and their microscopic uniformity (or aspect-ratio dependence), together with that of etch rate, selectivity, and damage. The precise control of $\mathrm{Si}$ etching in $\mathrm{Cl}$ - and $\mathrm{Br}$-based plasmas is indispensable for the fabrication of gate electrodes and shallow trench isolation of field effect transistors (FETs) ${ }^{1,2}$ through suppressing profile anomalies of sidewalls and bottom surfaces of the feature. ${ }^{6,7}$

Atomic- or nanometer-scale roughness on etched feature surfaces of Si has become an important issue to be resolved in the fabrication of nanoscale microelectronic devices, ${ }^{8-26}$ because the roughness formed during plasma etching would be comparable with the $\mathrm{CD}$ and the thickness of the layer being etched and/or the layer underlying; in addition, the

\footnotetext{
${ }^{a}$ Electronic mail: nakazaki.nobuya.58x@st.kyoto-u.ac.jp

b) Present address: Center for Semiconductor Research \& Development, Toshiba Corporation Semiconductor \& Storage Products Company, Komukai Toshiba-cho, Saiwai-ku, Kawasaki 212-8583, Japan.

${ }^{c}$ Present address: Division of Systems Research, Faculty of Engineering, Yokohama National University, Tokiwadai, Hodogaya-ku, Yokohama 240-8501, Japan.

${ }^{\mathrm{d})}$ Electronic mail: ono@kuaero.kyoto-u.ac.jp
}

nanoscale roughness of etched surfaces of $\mathrm{SiO}_{2},{ }^{21,24,27,28}$ metal, ${ }^{22}$ metal oxide, ${ }^{22}$ photoresist, ${ }^{29-31}$ polymer/polymeric, ${ }^{26,32,33}$ and low dielectric constant (low- $k$ ) films ${ }^{21,27,34}$ has also be an issue of great interest similarly. In gate fabrication, the roughness on feature sidewalls is responsible for the line edge roughness (LER) and line width roughness (LWR), ${ }^{35,36}$ which cause the variability in gate or channel length, and thus lead to that in transistor performance. ${ }^{37,38}$ Moreover, in advanced three-dimensional (3D) device structures, such as fin-type FETs (FinFETs), ${ }^{5,37,39}$ the effects of the fin as well as the gate LER and LWR become significant, ${ }^{37}$ because the LER and LWR occur also in fin etch processes, ${ }^{5,39}$ and the conducting channel of FinFETs is formed on top and sidewall surfaces of the fin. The surface roughness at the feature bottom affects the uniformity of bottom surfaces, which is responsible for a recess and damage in substrates, ${ }^{40}$ and in turn, also leads to the variability in transistor performance.

The surface roughness formed during plasma etching of $\mathrm{Si}$ has also been an important issue in MEMS fabrication processes, although the device dimensions are much larger than those of ULSI devices. In practice, the surface roughness is appreciated to affect the performance of MEMS through the fracture strength of fabricated microstructures ${ }^{41}$ and the friction force between moving microparts. ${ }^{42}$ Additionally, the processing time for etching deep microstructures is significantly long as compared with that in ULSI processes, which in turn would lead to the growth of more significant surface roughness in MEMS processes. 
Several experiments have been concerned with the formation and evolution of surface roughness during etching of blank (or planar) $\mathrm{Si}$ substrates in $\mathrm{SF}_{6},{ }^{9,17,20,23,26} \mathrm{CF}_{4} / \mathrm{O}_{2},{ }^{14}$ $\mathrm{Cl}_{2},{ }^{8,11,22}$ and $\mathrm{Ar}$ (Ref. 21) plasmas, and also in $\mathrm{XeF}_{2}$ gases with ${ }^{18}$ and without ${ }^{12} \mathrm{Ar}^{+}$ion beams. Correspondingly, several theoretical and/or numerical studies have been made to interpret the roughness observed in plasma etching experiments, using a continuum model, ${ }^{13-16}$ Monte Carlo simulation, ${ }^{15,16,19,20,24,26}$ and classical molecular dynamics (MD) simulation. ${ }^{10,25}$ The low-level surface roughening at the initial stage of plasma exposure is assumed to be caused by the noise (or stochastic roughening due to the uniformity of incident fluxes of ions and/or neutral etchants on surfaces at microscale) ${ }^{13-16,21,24}$ however, it is not sufficient for interpreting the following evolution of roughness, which has further invoked a few qualitative mechanisms, such as geometrical shadowing, ${ }^{13}$ surface reemission of etchants, ${ }^{14-16,19}$ and effects of etch inhibitors. ${ }^{20,26}$ More generic 3D Monte Carlo simulations have recently been performed to reproduce the surface features roughened in $\mathrm{Ar}$ (Ref. 24) and $\mathrm{SF}_{6}$ (Ref. 26) plasma etching of $\mathrm{Si}$, taking into account surface chemistries, such as sputtering and ion-enhanced etching with inhibitor deposition. However, the mechanisms for surface roughening during plasma etching are not yet fully understood, depending on a number of factors in processing.

We have recently investigated the formation and evolution of nanoscale surface roughness during plasma etching of $\mathrm{Si}$ in Cl-based plasmas by developing a 3D atomic-scale cellular model (ASCeM-3D) for simulating plasma-surface interactions and the resulting feature profile evolution during plasma etching. ${ }^{43-45}$ The ASCeM is our own semi-empirical, Monte Carlo-based simulation model with the cell removal method for interface evolution, and the ASCeM-3D revealed the surface roughening and ripple formation in response to ion incidence angle $\theta_{i}$ onto substrate surfaces. ${ }^{44,45}$ Preliminary experiments were also conducted for plasma etching of $\mathrm{Si}$ in $\mathrm{Cl}_{2}$, using an inductively coupled plasma (ICP) reactor, where a comparison of the etch rate (ER) and root-mean-square (rms) surface roughness was made with the ASCeM-3D simulations with $\theta_{i}=0^{\circ}$ (normal ion incidence) to verify the validity of our ASCeM-3D model. ${ }^{45}$

This paper presents more details of the experiments concerned with the nanoscale surface roughening during $\mathrm{Si}$ etching in ICP $\mathrm{Cl}_{2}$ plasmas, with emphasis being placed on a better understanding of plasma-surface interactions that are responsible for. Experiments were performed as a function of rf bias power by varying the feed gas flow rate, wafer stage temperature, and etching or plasma exposure time, including several plasma and surface diagnostics. The roughness of etched surfaces was quantified by the rms roughness and also in some cases by the power spectral density (PSD) distribution to gain information relative to both vertical and lateral extent of it. We observed two modes of surface roughening during plasma etching, roughening and smoothing (or non-roughening) modes, depending on bias power or ion incident energy onto substrate surfaces. The roughening mode may be unavailable in industry. However, studying the mechanisms responsible for the roughening as well as smoothing modes is important in industry as well as in research, because of the following two reasons: a better understanding of the mechanisms for the roughening mode is indispensable for finding the way how to suppress and/or control the evolution of the surface roughness during plasma exposure, because the roughening mode may be used unconsciously in industry as well as in research. The roughening mode may also be used, in some cases, to positively obtain roughed surfaces, or to obtain surface nanostructures, such as nanotextures, nanograsses, nanopillars, and nanocolumns. ${ }^{26}$ Section II describes the apparatus, operation, sample, and diagnostics used in this study. Then, Sec. III gives the results and discussion of the etching, together with those of the plasma and surface characterization concerned. A comparison is made between the experiments and ASCeM3D simulations, where some MD simulations for $\mathrm{Si} / \mathrm{Cl}$ and $\mathrm{Si} / \mathrm{SiCl}$ systems are invoked to further understand the atomistic mechanisms concerned with ionized etch products or silicon chloride ions. Finally, Sec. IV summarizes conclusions of this paper.

\section{EXPERIMENT}

Figure 1 shows the experimental setup. The ICP plasma reactor consisted of a stainless-steel chamber $25 \mathrm{~cm}$ in diameter and $30 \mathrm{~cm}$ high, a three-turn planar coil on a dielectric window at the top, and a 4-in.-diameter electrode or wafer stage at the bottom; the distance between the dielectric window and the wafer was $\sim 9 \mathrm{~cm}$ in experiments, and the effective volume of the chamber was $V_{0} \approx 1.2 \times 10^{4} \mathrm{~cm}^{3}$. The discharge was established by $13.56-\mathrm{MHz}$ rf powers of nominally $P_{\mathrm{ICP}}=450 \mathrm{~W}$. The wafer stage was capacitively coupled to a 13.56-MHz rf power supply through an impedance matching network for additional rf biasing, where the rf bias power was varied in the range $P_{\mathrm{rf}}=0-200 \mathrm{~W}$ to vary the ion incident energy $E_{i}$ onto substrate surfaces. The reactor chamber was pumped down to a base pressure $<5 \times 10^{-7}$ Torr by a $1000 \mathrm{l} / \mathrm{s}$ turbomolecular pump, and pure $\mathrm{Cl}_{2}$ gases was introduced therein with the flow rate being varied in the range $F_{0}=5-50 \mathrm{sccm}$ (typically $20 \mathrm{sccm}$ ). In these experiments, the pressure was kept constant at $P_{0}=20 \mathrm{mTorr}$

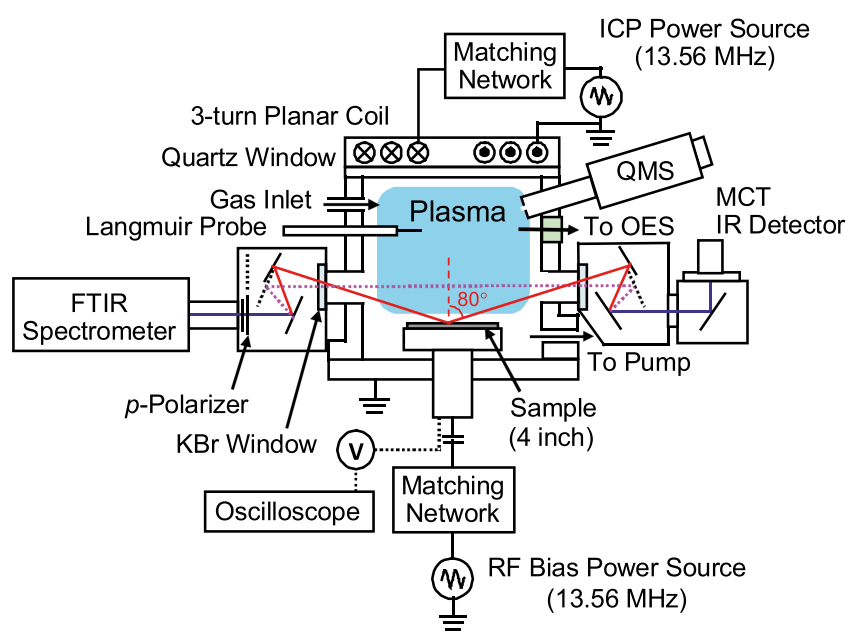

FIG. 1. Experimental setup for plasma etching using an ICP plasma reactor, including several plasma and surface diagnostics employed. 
$(2.66 \mathrm{~Pa})$ by adjusting the pumping rate with an automatic throttle valve between the chamber and the tubomolecular pump, where the gas residence time in the reactor chamber was estimated to be of the order of $\tau_{r}=P_{0} V_{0} / F_{0} \approx 3.7-0.37 \mathrm{~s}$ (typically $0.9 \mathrm{~s}$ ).

Samples for etching were 4-in.-diameter blank $\operatorname{Si}(100)$ wafers of $n$-type with a resistivity of $\rho_{r} \approx 10$ and $0.02 \Omega \cdot \mathrm{cm}$, which were pasted with silicone high vacuum grease into place on the wafer stage that was temperature controlled in the range $T_{s}=20-200^{\circ} \mathrm{C}$ (typically $20^{\circ} \mathrm{C}$ ). The samples were cleaned through $\mathrm{HF}$ acid dipping followed by deionized water rinsing, prior to experiments. A brief pre-etch breakthrough step (nominally $10 \mathrm{~s}$ at $P_{\mathrm{rf}}=30 \mathrm{~W}$ ) was employed prior to main etch, to remove native and/or chemical oxide layers on $\mathrm{Si}$; in practice, at low $P_{\mathrm{rf}}<5 \mathrm{~W}$, the etching of $\mathrm{Si}$ did not occur without the breakthrough step, owing to oxide layers thereon. The etched depth was measured by stylus profilometry (Veeco Dektak $6 \mathrm{M}$ ), and the etch rate was calculated by dividing the etched depth by the etching time, which was varied in the range $t_{\text {etch }}=1-20$ min (typically $2 \mathrm{~min}$ ). Moreover, the etched surfaces were examined by using atomic force microscopy (AFM, Shimadzu SPM-9600) to measure the rms surface roughness $(\sim 0.15 \mathrm{~nm}$ after wafer wet cleaning prior to experiments), and also by scanning electron microscopy (SEM, JEOL JSM-7500FA) to take the corresponding surface images. These surface diagnostics were performed with no wafer cleaning after etching, and 1000-nm-thick thermally grown $\mathrm{SiO}_{2}$ films on $\mathrm{Si}$ were also employed for reference in these experiments to measure the etch selectivity of $\mathrm{Si}$ over $\mathrm{SiO}_{2}$.

Several plasma diagnostics were also employed to characterize the plasma at around the wafer position during processing, using optical emission spectroscopy (OES), quadrupole mass spectrometry (QMS), and Langmuir probe measurement. The OES employed a $10-\mathrm{cm}$ focal length spectrometer (Ocean Optics HR2000CG-UV-NIR with a grating of 300 lines $/ \mathrm{mm}$ and a spectral resolution of $1 \mathrm{~nm}$ ), where the optical emission spectra of the plasma were observed through a quartz window attached on the chamber sidewalls. The QMS employed a quadrupole mass spectrometer with an electrostatic energy analyzer (Hiden HAL EQP 500 with a mass range of $m / e=1-500 \mathrm{amu}$ ); the system was differentially pumped down to a base pressure $<1 \times 10^{-8}$ Torr, and was operated at $<5 \times 10^{-7}$ Torr in experiments, sampling a portion of the discharge through a $0.1-\mathrm{mm}$-diameter orifice near the periphery of the wafer stage at $\sim 3 \mathrm{~cm}$ above the substrate. The Langmuir probe had a cylindrical tungsten tip $0.5 \mathrm{~mm}$ in diameter and $10 \mathrm{~mm}$ long, which was positioned horizontally at $\sim 3 \mathrm{~cm}$ above the center of the substrate. In addition, the voltage waveform and thus the dc self-bias voltage $V_{\mathrm{dc}}$ at the wafer stage were routinely monitored by a voltage probe, which gave a measure of the ion incident energy $E_{i}=V_{p}-V_{\mathrm{dc}}$ onto substrates, taking into account the plasma potential $V_{p}$ measured by the Langmuir probe.

Moreover, as also shown in Fig. 1, Fourier transform infrared (FTIR) absorption spectroscopy was utilized to observe etch-related chemical species, such as Si-containing etch products and/or byproducts, in the plasma and also on substrate surfaces. ${ }^{46,47}$ The IR optical system consisted of an
FTIR spectrometer (Thermo Nicolet iS50R with a KBr beam splitter), which was reconfigured for the probe beam to pass through the reactor chamber located outside of the optical bench of the instrument; the system was being purged with dry nitrogen gases to remove the residual carbon dioxide and water vapor therein. In transmission absorption spectroscopy (TAS) for observation of species in the gas phase, the IR beam from the FTIR bench was directed through 4-cm-diameter $\mathrm{KBr}$ optical flats into the chamber, $\sim 3 \mathrm{~cm}$ above the substrate parallel to its surface. The TAS spectra were observed in single-pass operation with an absorption path length of $l$ $\approx 25 \mathrm{~cm}$ in the chamber, and changes of the IR beam intensity due to the absorption were detected with a liquid-nitrogen-cooled MgCdTe (MCT) detector placed $\sim 40 \mathrm{~cm}$ apart from the exit $\mathrm{KBr}$ window. In reflection absorption spectroscopy (RAS) for observation of species on the surface, the IR beam from the FTIR bench was passed through a thalliumbromide-iodide (KRS-5) polarizer, and the $p$-polarized beam was then focused onto substrate surfaces, where the RAS spectra were observed in single reflection mode at an incident angle of $80^{\circ}$.

In both of these observations, the FTIR data were recorded in the wavenumber range $4000-450 \mathrm{~cm}^{-1}$ at a resolution of 2 or $4 \mathrm{~cm}^{-1}$ by averaging over 250-500 scans (taking $4-8 \mathrm{~min}$ ), and the Happ-Genzel apodization was applied to the interferogram before carrying out the Fourier transform. Background or reference spectra were collected in the presence of feed gas flows with a wafer being in place on the stage; the sample spectra were then taken with the discharge switched on (or during etching), where the noise level in the spectra was not affected seriously by operating the discharge. The absorbance spectrum was determined by calculating $-\ln \left(I / I_{0}\right)$ as a function of wavenumber, where $I$ and $I_{0}$ are the IR beam intensities at the detector corresponding to the sample and reference spectra, respectively.

\section{RESULTS AND DISCUSSION}

\section{A. Plasma parameters}

Figure 2 shows the plasma parameters as a function of $\mathrm{rf}$ bias power in the range $P_{\mathrm{rf}}=0-200 \mathrm{~W}$, measured by Langmuir probes during ICP plasma etching of $\mathrm{Si}$ in $\mathrm{Cl}_{2}$ under typical operating conditions of $P_{\mathrm{ICP}}=450 \mathrm{~W}, P_{0}=20 \mathrm{mTorr}$, $F_{0}=20 \mathrm{sccm}$, and $T_{s}=20^{\circ} \mathrm{C}$ : electron density $n_{e}$ and temperature $T_{e}$, positive ion density $n_{i}$, plasma potential $V_{p}$, and potential difference $V_{p}-V_{\mathrm{dc}}$. The probe data were analyzed assuming an effective ion mass $m_{i, \text { eff }}=\sum_{j} \chi_{j} m_{j}$, where the concentration fraction $\chi_{j}$ of the ion species $j$ with a mass $m_{j}$ was determined by QMS for each condition. The results indicate that the ion $n_{i}$ and electron $n_{e}$ densities remain almost unchanged over the $P_{\mathrm{rf}}$ range investigated, while the electron temperature $T_{e}$ increases slightly with increasing $P_{\mathrm{rf}}$. The corresponding ion flux concerned (or the ion saturation current to the probe measured) remains almost constant at $\Gamma_{i}{ }^{0}=0.61$ $n_{i}\left(k_{B} T_{e} / m_{i, \mathrm{eff}}\right)^{1 / 2} \approx(0.5-0.6) \times 10^{16} \mathrm{~cm}^{-2} \mathrm{~s}^{-1},{ }^{48}$ where $k_{B}$ is the Boltzmann constant. Moreover, the plasma potential $V_{p}$ remains almost unchanged at low $P_{\text {rf }}$, while it increases slightly with increasing $P_{\mathrm{rf}}>50 \mathrm{~W}$, corresponding to an increase in $T_{e}$. The potential difference $V_{p}-V_{\mathrm{dc}}$ increases 

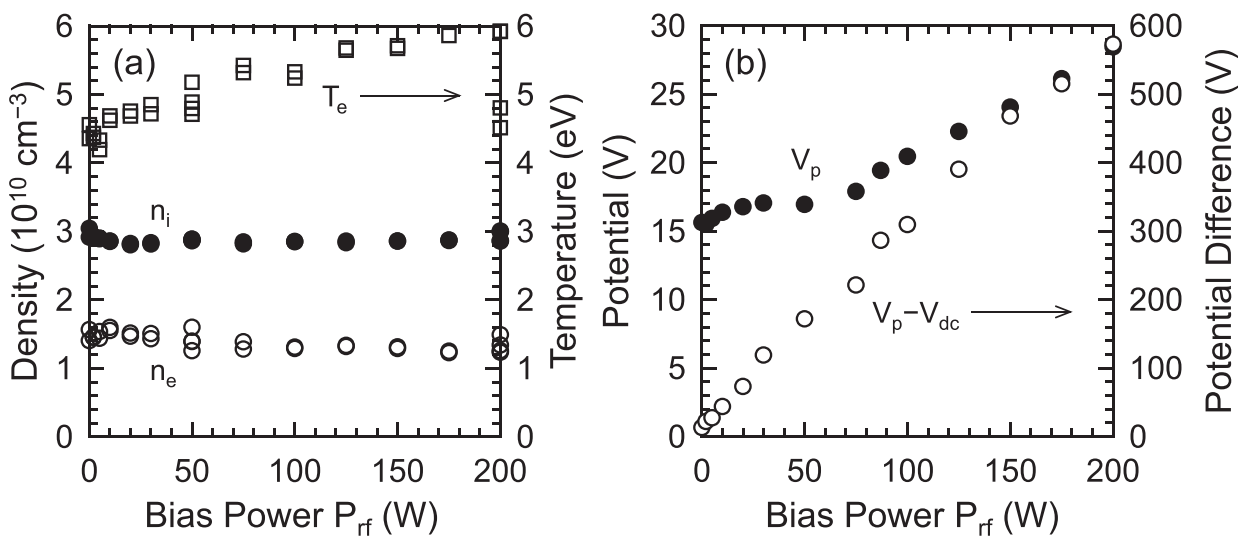

FIG. 2. Plasma parameters as a function of $\mathrm{rf}$ bias power $P_{\mathrm{rf}}$, measured by Langmuir probes during ICP plasma etching of $\mathrm{Si}$ in $\mathrm{Cl}_{2}$ under typical operating conditions of $P_{\mathrm{ICP}}=450 \mathrm{~W}$, $P_{0}=20 \mathrm{mTorr}, F_{0}=20 \mathrm{sccm}$, and $T_{s}=20^{\circ} \mathrm{C}$ : (a) electron density $n_{\mathrm{e}}$ and temperature $T_{\mathrm{e}}$, and positive ion density $n_{i}$; (b) plasma potential $V_{p}$ and potential difference $V_{p}-V_{\mathrm{dc}}$, where $V_{\mathrm{dc}}$ is the dc self-bias voltage at the wafer stage, and $E_{i}=V_{p}-V_{\mathrm{dc}}$ is a measure of the ion incident energy onto substrate surfaces.

with increasing $P_{\mathrm{rf}}$, owing to the dc self-bias voltage $V_{\mathrm{dc}}$ decreased, giving an ion incident energy in the range $E_{i} \approx 13-570 \mathrm{eV}$ for $P_{\mathrm{rf}}=0-200 \mathrm{~W}$. It is noted here that the potential $V_{p}$ was consistent with the ion energy distribution measured by QMS (not shown), and that the probe data did not depend significantly on gas flow rate $F_{0}$ and stage temperature $T_{s}$, as well as on the wafer resistivity.

\section{B. Etch rate and surface roughness}

Figure 3 shows typical AFM images of the Si surfaces etched in ICP $\mathrm{Cl}_{2}$ plasmas for $t_{\text {etch }}=2 \mathrm{~min}$ with four different ion incident energies of $E_{i} \approx 22-470 \mathrm{eV}$ under the same conditions as in Fig. 2. Inset is an image of wet-cleaned $\mathrm{Si}$ surfaces prior to etching $\left(t_{\mathrm{etch}}=0\right)$ for reference. These surface images and the data on the rms surface roughness were acquired in tapping mode on a scan area of $1 \times 1 \mu \mathrm{m}^{2}$ with the resolution of $256 \times 256$ pixels and a scan rate of $1 \mathrm{~Hz}$, using a microfabricated $\mathrm{Si}$ cantilever with a tip radius less

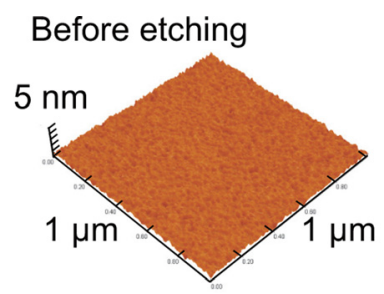

(a) $E_{i}=22 \mathrm{eV}$

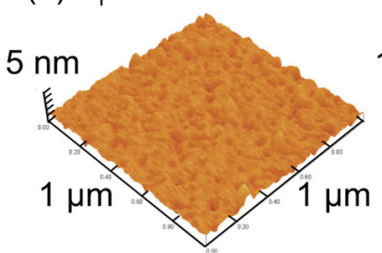

(c) $E_{i}=220 \mathrm{eV}$

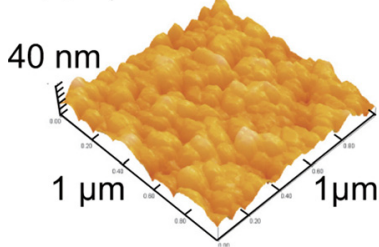

(b) $E_{i}=120 \mathrm{eV}$

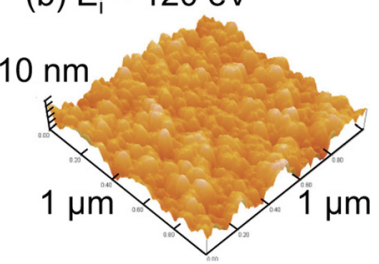

(d) $E_{i}=470 \mathrm{eV}$

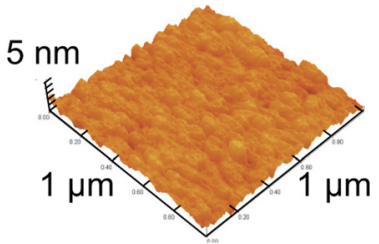

FIG. 3. Typical AFM images of the Si surfaces etched in $\mathrm{ICP} \mathrm{Cl}_{2}$ plasmas for $t_{\text {etch }}=2$ min with four different ion incident energies of $E_{i} \approx$ (a) 22, (b) 120, (c) 220, and (d) $470 \mathrm{eV}$ under the same conditions as in Fig. 2. Inset is an image of wet-cleaned Si surfaces prior to etching for reference. than $10 \mathrm{~nm}$. The AFM images indicate that as the bias power $P_{\text {rf }}$ or ion energy $E_{i}$ is increased, the roughness of etched $\mathrm{Si}$ surfaces increases and then decreases.

Figure 4 shows the etch rates of $\mathrm{Si}$ and $\mathrm{SiO}_{2}$ and the rms surface roughness of $\mathrm{Si}$ as a function of ion incident energy in the range $E_{i} \approx 22-520 \mathrm{eV}$, obtained in $\mathrm{ICP} \mathrm{Cl}_{2}$ plasma etching $\left(t_{\text {etch }}=2 \mathrm{~min}\right)$ for four different gas flow rates of $F_{0}=5,10,20$, and $50 \mathrm{sccm}$ under otherwise the same conditions as in Fig. 2. The error bars (shown typically for $F_{0}=20$ $\mathrm{sccm}$ ) represent the variation in the raw data for more than ten etching experiments using sample wafers with different as well as similar resistivities and cleaning recipes. Note that the experiments for $F_{0}=5 \mathrm{sccm}$ were restricted to low $E_{i}<120 \mathrm{eV}$, because the present system with $P_{0}=20 \mathrm{mTorr}$ and $F_{0}=5 \mathrm{sccm}$ starves for feed gases at higher $E_{i}$, owing to increased $\mathrm{Si}$ etch rates; in practice, the amount of Si etched per minute is estimated to be $\left(\rho_{s} \mathrm{ER}\right) S \approx 8.1 \times 10^{19}$ atoms/ $\min$ for an $E R=200 \mathrm{~nm} / \mathrm{min}$ of a 4-in.-diameter wafer, corresponding to an inflow rate $\approx 3.0 \mathrm{sccm}(1 \mathrm{sccm}=2.686$ $\times 10^{19}$ atoms or molecules/min at standard conditions), where $\rho_{s}$ is the $\mathrm{Si}$ atom density and $S$ is the surface area of substrates. The results indicate that for any $F_{0}$, as the bias power $P_{\mathrm{rf}}$ or ion energy $E_{i}$ is increased, the $\mathrm{Si}$ and $\mathrm{SiO}_{2}$ etch rates increase, and the etch selectivity of $\mathrm{Si}$ over $\mathrm{SiO}_{2}$ decreases; on the other hand, the rms roughness of etched $\mathrm{Si}$ surfaces increases, peaks at around $E_{i}=E_{p} \approx 200-300 \mathrm{eV}$ (depending slightly on $F_{0}$ ), and then decreases substantially down to a low level $<0.4 \mathrm{~nm}$. Thus, it follows that in these experiments, the roughness scales with the etch rate at low $E_{i}<E_{p}$, while it does not scale with the etch rate at higher $E_{i}>E_{p}$. The increase followed by the decrease in surface roughness with increasing $P_{\mathrm{rf}}$ or $E_{i}$ has so far not been observed in plasma etching experiments of $\mathrm{Si}$ : some experiments showed an increase in roughness as well as etch rate with $P_{\mathrm{rf}}$ in $\mathrm{Cl}_{2}$ plasma; ${ }^{11}$ some other experiments showed a decrease in roughness with $P_{\mathrm{rf}}$ in $\mathrm{SF}_{6}$ plasma, ${ }^{9}$ with $E_{i}$ in the range $20-100 \mathrm{eV}$ in $\mathrm{SF}_{6}$ plasma, ${ }^{23}$ and with $E_{i}$ in the range $100-250 \mathrm{eV}$ in $\mathrm{Cl}_{2}$ plasma, ${ }^{22}$ in contrast to an increase in etch rate.

Moreover, at a given $P_{\mathrm{rf}}$ or $E_{i}$ in Fig. 4, the $\mathrm{Si}$ etch rate and the selectivity tend to be slightly increased for increased flow rates $F_{0}$, while the $\mathrm{SiO}_{2}$ etch rate does not depend significantly on $F_{0}$, as has been known to be due to reduced concentrations of etch products/byproducts in the plasma 

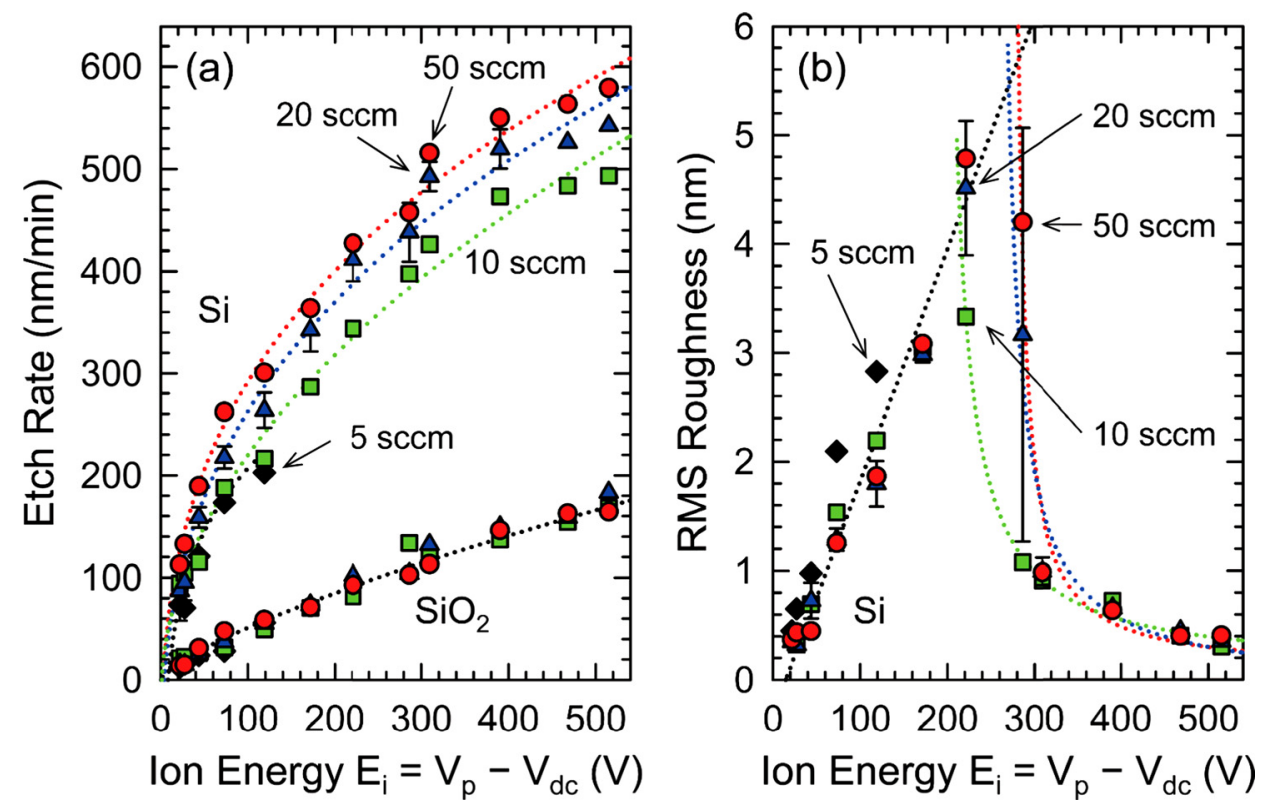

FIG. 4. (a) Etch rates of $\mathrm{Si}$ and $\mathrm{SiO}_{2}$ and (b) rms surface roughness of $\mathrm{Si}$ as a function of ion incident energy in the range $E_{i} \approx 22-520 \mathrm{eV}$, obtained in ICP $\mathrm{Cl}_{2}$ plasma etching $\left(t_{\text {etch }}=2 \mathrm{~min}\right)$ for four different gas flow rates of $F_{0}=5$, 10,20 , and $50 \mathrm{sccm}$ under otherwise the same conditions as in Fig. 2. The rms roughness shown is that measured for the AFM scan area of $1 \times 1 \mu \mathrm{m}^{2}$ as in Fig. 3, and the dotted lines are for guiding the eyes only. Note that the experiments for $F_{0}=5 \mathrm{sccm}$ were restricted to low $E_{i}<120 \mathrm{eV}$, because the present system with $P_{0}=20 \mathrm{mTorr}$ and $F_{0}=5 \mathrm{sccm}$ starves for feed gases at higher $E_{i}$, owing to increased Si etch rates.

during high-gas-flow-rate or short-gas-residence-time plasma etching of $\mathrm{Si}^{49}$ On the other hand, the rms surface roughness of $\mathrm{Si}$ appears to be almost independent of $F_{0}$ at low $E_{i}<200 \mathrm{eV}$ and high $E_{i}>300 \mathrm{eV}$, although the ion energy $E_{p}$ giving a peak of the rms roughness, together with its peak value, tends to be slightly increased for increased $F_{0}$.

Figure 5 shows the etch rates of $\mathrm{Si}$ and $\mathrm{SiO}_{2}$ as a function of the square root $\sqrt{E_{i}}$ of the ion incident energy for four different gas flow rates $F_{0}$, which are the data replotted from the preceding Fig. 4(a). The results indicate that for any $F_{0}$, both the $\mathrm{Si}$ and $\mathrm{SiO}_{2}$ etch rates are approximately proportional to $\sqrt{E_{i}}$, which reflects the universal energy dependence of the yield for ion bombardment-induced etch

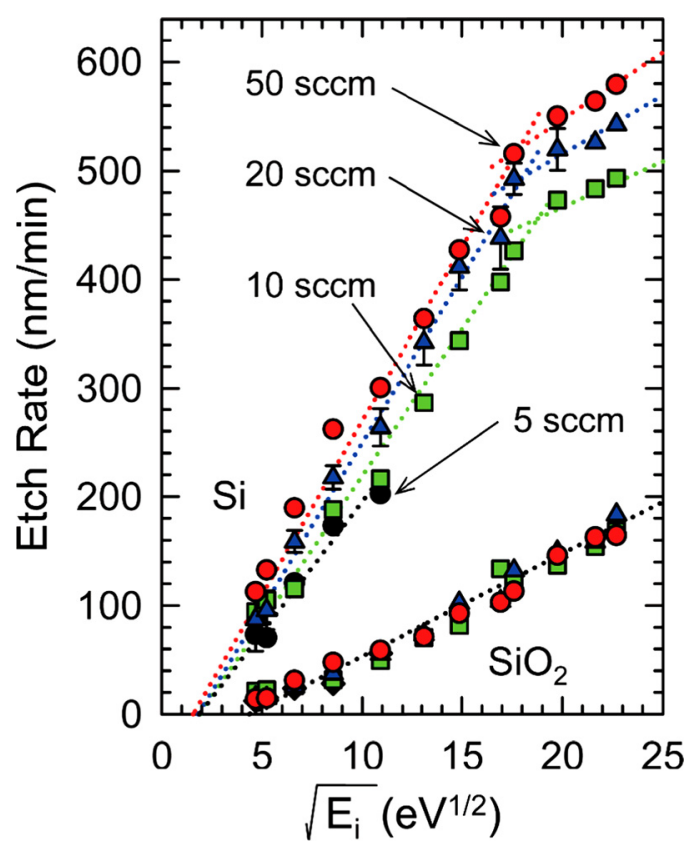

FIG. 5. Etch rates of $\mathrm{Si}$ and $\mathrm{SiO}_{2}$ as a function of the square root $\sqrt{E_{i}}$ of the ion incident energy for four different gas flow rates $F_{0}$, which are the data replotted from the preceding Fig. 4(a). The dotted lines are linear fits to guide the eyes. processes, such as physical sputtering and ion-enhanced etching: ${ }^{50-52} Y=\rho_{s} \mathrm{ER} / \Gamma_{i}^{0}=A\left(E_{i}{ }^{1 / 2}-E_{\mathrm{th}}^{1 / 2}\right)$, where the yield $Y$ is defined as the total number of $\mathrm{Si}$ atoms desorbed from substrate surfaces per ion impact, the $\mathrm{Si}$ atom density of substrates is $\rho_{s} \approx 5.0$ and $2.2 \times 10^{22} \mathrm{~cm}^{-3}$ for $\mathrm{Si}$ and $\mathrm{SiO}_{2}$, respectively, ${ }^{53} \Gamma_{i}^{0}$ is the ion flux incident on substrate surfaces, $A$ is a proportional constant or scaling factor, and $E_{\text {th }}$ is the threshold energy concerned. The liner dependence of the yield $Y$ on $\sqrt{E_{i}}$ implies that the etching is limited by the momentum transfer from energetic ions to substrate atoms through the collision-cascade mechanism. ${ }^{50-52}$ In the present experiments, the ion flux $\Gamma_{i}^{0} \approx(0.5-0.6)$ $\times 10^{16} \mathrm{~cm}^{-2} \mathrm{~s}^{-1}$ does not change significantly with $E_{i}$ (as mentioned earlier with respect to Fig. 2), and so the etch rate ER should also be proportional to $\sqrt{E_{i}}$. The threshold is estimated to be $E_{\mathrm{th}} \approx 4$ and $20 \mathrm{eV}$ for $\mathrm{Si}$ and $\mathrm{SiO}_{2}$, respectively, by extrapolating the ER to zero, being almost independent of flow rate $F_{0}$ and in agreement with previous plasma etching experiments in $\mathrm{Cl}_{2} .{ }^{54}$ Moreover, the $\mathrm{Si}$ etch yield is estimated to be typically $Y \approx 1.3-7.3(\mathrm{ER} \approx 80-440 \mathrm{~nm} / \mathrm{min})$ at ion energies in the rage $E_{i} \approx 20-300 \mathrm{eV}$, giving the factor $A \approx 0.46$ in $\mathrm{eV}^{-1 / 2}$, where the $Y$ and thus $A$ depends slightly on $F_{0}$ in these experiments. The $A$ factor presently estimated compares relatively well with previous plasma etching experiments of $\mathrm{Si}$ in $\mathrm{Cl}_{2}$ plasmas $(A \approx 0.275$ in Ref. 55, and 0.353 in Ref. 56). In addition, the $\mathrm{SiO}_{2}$ etch yield is estimated to be $Y \approx 0.11-1.2 \quad(\mathrm{ER} \approx 15-160 \mathrm{~nm} / \mathrm{min})$ at $E_{i} \approx 20-500 \mathrm{eV}$, being almost independent of $F_{0}$.

It is further noted in Fig. 5 that for any $F_{0}$, the curve of ER or $Y$ versus $\sqrt{E_{i}}$ for $\mathrm{Si}$ exhibits a transition point at around $E_{i} \approx 300-400 \mathrm{eV}$, above which the $Y$ or ER increases more slowly with increasing $E_{i}$, although it remains nearly proportional to $\sqrt{E_{i}}$; in practice, the slope or proportional constant $A$ of the curve is estimated to decrease by about a factor of 2-3 above the transition point, implying some change in reaction kinetics on surfaces being etched. A comparison between Figs. 4(b) and 5 indicates that for $\mathrm{Si}$, the ion energy $E_{p}$ giving the peak rms roughness corresponds 


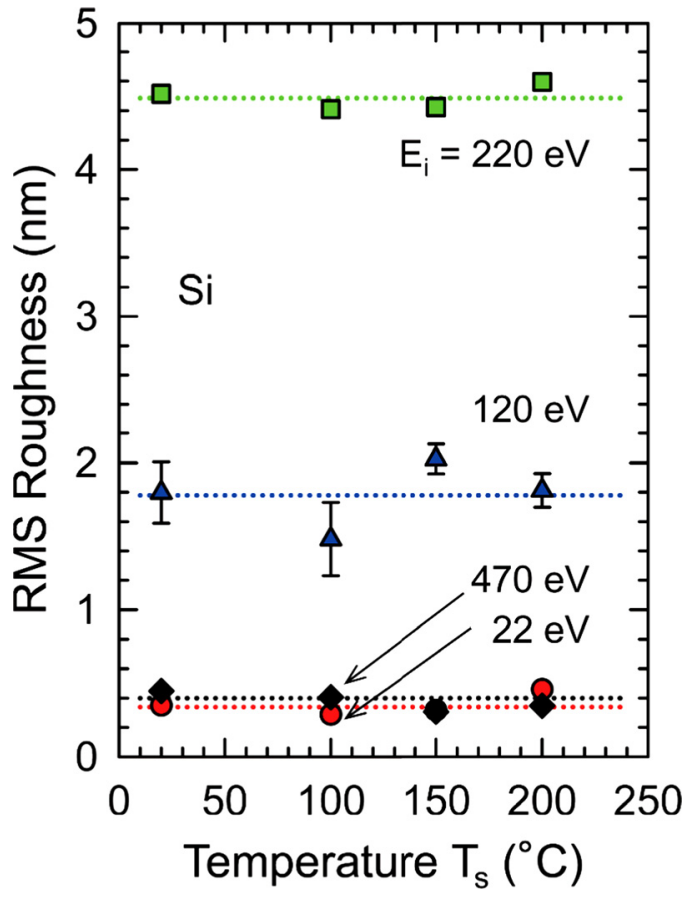

FIG. 6. RMS surface roughness of $\mathrm{Si}$ as a function of wafer stage temperature in the range $T_{s}=20-200^{\circ} \mathrm{C}$, obtained under otherwise the same conditions as in Fig. 2, in ICP $\mathrm{Cl}_{2}$ plasma etching $\left(t_{\text {etch }}=2 \mathrm{~min}\right.$ ) for four different ion incident energies of $E_{i} \approx 22-470 \mathrm{eV}$. The dotted lines are for guiding the eyes only.

roughly to the transition point for the $Y$ or ER versus $\sqrt{E_{i}}$ curve, although the latter tends to be slightly decreased for increased flow rates $F_{0}$, and the latter is a little higher than the former by about $\Delta E_{i} \approx 50-150 \mathrm{eV}$.

Figure 6 shows the rms surface roughness of $\mathrm{Si}$ as a function of wafer stage temperature in the range $T_{s}$ $=20-200{ }^{\circ} \mathrm{C}$, obtained under otherwise the same conditions as in Fig. 2, in ICP $\mathrm{Cl}_{2}$ plasma etching $\left(t_{\text {etch }}=2 \mathrm{~min}\right)$ for four different ion incident energies of $E_{i} \approx 22-470 \mathrm{eV}$. The error bars (shown typically for $E_{i} \approx 120 \mathrm{eV}$ ) have the same properties as those in Fig. 4. The results indicate no significant $T_{s}$ dependence of the roughness of etched Si surfaces, as well as the $\mathrm{Si}$ and $\mathrm{SiO}_{2}$ etch rates (not shown), although the temperature $T_{s}$ is expected to modify the surface reaction kinetics during etching. No $T_{\mathrm{s}}$ dependence of both the roughness and etch rate has been observed in plasma etching experiments of $\mathrm{Si}$ in $\mathrm{SF}_{6}$ for $T_{s} \approx-100-40{ }^{\circ} \mathrm{C}{ }^{9}$ This may be attributed partly to the wafer surface temperature that is often different from the stage temperature $T_{\mathrm{s}}$ (usually measured by a thermocouple attached to the stage) during plasma etching; in practice, the surface temperature during etching was measured to be higher than $T_{s}$ by using FTIR-RAS (increasing with increasing $P_{\mathrm{rf}}$ or $\left.E_{i}\right){ }^{57}$

Figure 7 shows the rms surface roughness of $\mathrm{Si}$ as a function of etching or plasma exposure time in the range $t_{\text {etch }}=1-20 \mathrm{~min}$, obtained in ICP $\mathrm{Cl}_{2}$ plasma etching for seven different ion incident energies of $E_{i} \approx 22-520 \mathrm{eV}$ under the same conditions as in Fig. 2. The error bars (shown typically for $E_{i} \approx 220 \mathrm{eV}$ ) have the same properties as those in Fig. 4. Typical AFM images of the Si surfaces etched for $t_{\text {etch }}=20 \mathrm{~min}$ are also shown in Fig. 8 for reference, which are compared with those for $t_{\text {etch }}=2 \mathrm{~min}$ in Fig. 3 . The results indicate that at low $E_{i}<E_{p}\left(\approx 250 \mathrm{eV}\right.$ for $F_{0}$ $=20 \mathrm{sccm}$ ), the rms roughness of etched surfaces increases almost linearly with time during etching, where the higher the $E_{i}$, the larger is the increase in roughness; in particular, at $E_{i} \approx 220 \mathrm{eV}$, the rms roughness reaches an extraordinarily large value of $>70 \mathrm{~nm}$ at $t=20 \mathrm{~min}$. In contrast, at higher $E_{i}>E_{p}$, the roughness tends to reach quasi-steady state after some increase at the initial stage of etching $(t<1 \mathrm{~min})$, where the higher the $E_{i}$, the smaller is the roughness at steady state; in particular, at $E_{i}>470 \mathrm{eV}$, the rms roughness remains at a low level $<0.4 \mathrm{~nm}$, which is only a little larger than the initial value of $\sim 0.15 \mathrm{~nm}$ prior to etching. It is noted here that over the $P_{\text {rf }}$ or $E_{i}$ range investigated, the etched depth increased almost linearly with time, or the etch rate reached quasi-steady state soon after the start of etching (not shown); thus, it follows that in these experiments, the roughness evolution does not follow that of the etch rate at low
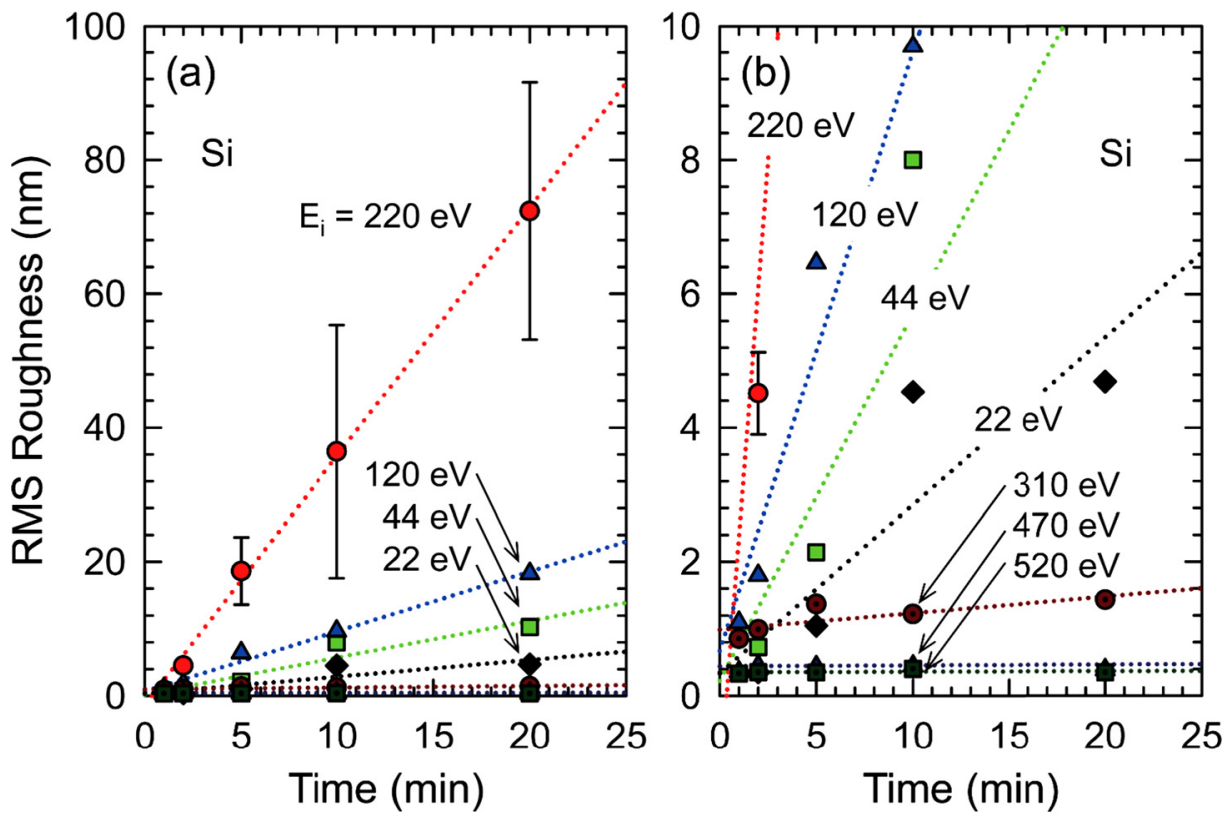

FIG. 7. RMS surface roughness of $\mathrm{Si}$ as a function of etching or plasma exposure time in the range $t_{\text {etch }}=1-20$ min, obtained in ICP $\mathrm{Cl}_{2}$ plasma etching for seven different ion incident energies of $E_{i} \approx 22-520 \mathrm{eV}$ under the same conditions as in Fig. 2. The vertical scale (rms roughness) of the figure ranges from (a) 0 to $100 \mathrm{~nm}$ and (b) 0 to $10 \mathrm{~nm}$. The dotted lines are for guiding the eyes only. 
(a) $E_{i}=22 \mathrm{eV}$

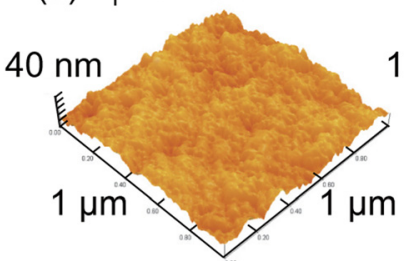

(b) $E_{i}=120 \mathrm{eV}$

(c) $E_{i}=220 \mathrm{eV}$

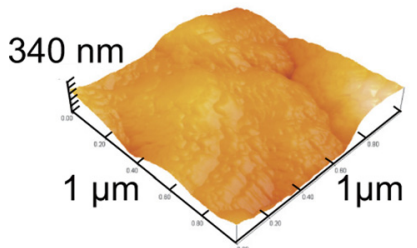

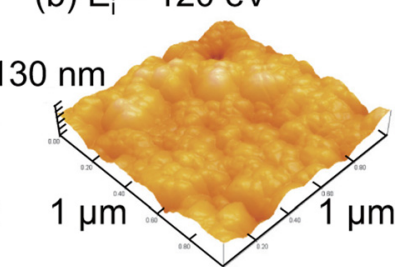

(d) $E_{i}=470 \mathrm{eV}$

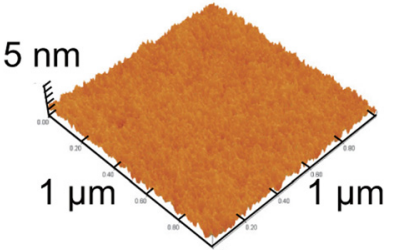

FIG. 8. Typical AFM images of the Si surfaces etched in $\mathrm{ICP} \mathrm{Cl}_{2}$ plasmas for $t_{\mathrm{etch}}=20 \mathrm{~min}$ with four different ion incident energies of $E_{i} \approx$ (a) 22 , (b) 120 , (c) 220 , and (d) $470 \mathrm{eV}$ under the same conditions as in Fig. 2. These images are compared with those for $t_{\text {etch }}=2 \mathrm{~min}$ in Fig. 3.

$E_{i}<E_{p}$. The increase in surface roughness with time during etching, along with quasi-steady etch rates, has been observed in several plasma etching experiments of $\mathrm{Si}$ in $\mathrm{SF}_{6}$ (Refs. 9, 20, 23, and 26) and $\mathrm{CF}_{4} / \mathrm{O}_{2},{ }^{14}$ while the low-level, quasi-steady roughness has not been reported so far.

Figure 9 shows typical SEM images of the time evolution of the $\mathrm{Si}$ surfaces etched in ICP $\mathrm{Cl}_{2}$ plasmas $\left(t_{\text {etch }}=1-20 \mathrm{~min}\right)$ with four different ion incident energies of $E_{i} \approx 22-470 \mathrm{eV}$ under the same conditions as in Fig. 2. Also shown are the images of wet-cleaned Si surfaces prior to etching $\left(t_{\text {etch }}=0\right)$ for reference. These SEM images were found to be consistent visually with the corresponding AFM images (e.g., Figs. 3 and 8 for $t_{\text {etch }}=2$ and $20 \mathrm{~min}$ ), and also with the data of the rms surface roughness measured therefrom [Figs. 4(b) and 7]: at low $E_{i}<E_{p}$ $(\approx 250 \mathrm{eV})$, the surface roughness continues to evolve with time, where the higher the $E_{i}$, the more significant is the evolution of roughness, and the roughness at $E_{i} \approx 220 \mathrm{eV}$

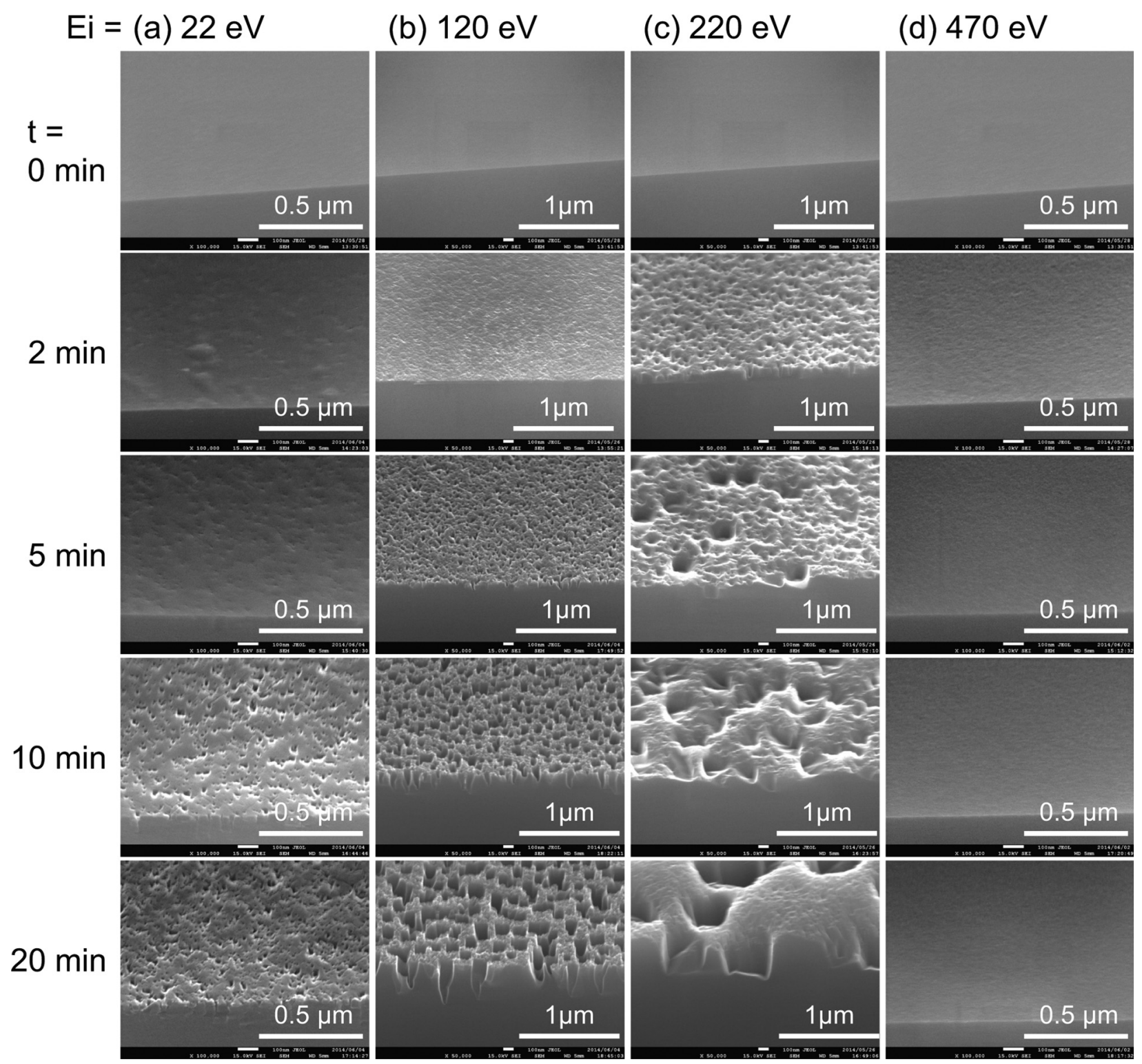

FIG. 9. Typical SEM images of the time evolution of the Si surfaces etched in $\mathrm{ICP} \mathrm{Cl}_{2}$ plasmas $\left(t_{\mathrm{etch}}=1-20\right.$ min) with four different ion incident energies of $E_{i} \approx($ a) 22, (b) 120, (c) 220, and (d) $470 \mathrm{eV}$ under the same conditions as in Fig. 2. Also shown are the images of wet-cleaned Si surfaces prior to etching $\left(t_{\mathrm{etch}}=0\right.$ ) for reference. 
becomes extraordinarily large in lateral as well as vertical extent at $t=20 \mathrm{~min}$. In contrast, at higher $E_{i}>E_{p}$, the roughness tends to reach quasi-steady state soon after the start of etching, remaining low at $E_{i}>470 \mathrm{eV}$. Thus, we may consider that in these experiments, the evolution of the rms roughness of etched surfaces shown in Fig. 7 fully reflects that of the degree of the surface roughness concerned, although the AFM is generally assumed to be no longer appropriate to measure a large rms roughness $>20 \mathrm{~nm}$.

Hence, it is concluded that there are two modes of surface roughening during plasma etching of $\mathrm{Si}$ in $\mathrm{Cl}_{2}$, which occur depending on $\mathrm{rf}$ bias power $P_{\mathrm{rf}}$ or ion incident energy $E_{i}$ : one is the roughening mode at low $E_{i}<E_{p}$ $(\approx 200-300 \mathrm{eV})$, where the rms roughness of etched surfaces increases with increasing $E_{i}$, scaling with the etch rate (Fig. 4 ), and the etch rate ER or etch yield $Y$ versus $\sqrt{E_{i}}$ curve exhibits a relatively large slope or proportional constant (Fig. 5); in this mode, the rms roughness increases almost linearly with time during etching (Fig. 7), while the etch rate reaches quasi-steady state soon after the start of etching. The other is the smoothing or non-roughening mode at higher $E_{i}>E_{p}$, where the rms surface roughness decreases substantially with increasing $E_{i}$ down to a low level at $E_{i} \gg$ $E_{p}$, not scaling with the etch rate (Fig. 4), and the ER or $Y$ versus $\sqrt{E_{i}}$ curve exhibits a decreased proportional constant (Fig. 5); in this mode, the rms roughness tends to reach quasi-steady state after some increase at the initial stage of etching (Fig. 7), where the roughness evolution follows that of the etch rate in a sense. In the present experiments, the transition from roughening to smoothing modes is sharp with increasing $P_{\mathrm{rf}}$ or $E_{i}$; and the transition point $E_{p}$ for the rms roughness versus $E_{i}$ curve appears to correspond to that for the ER or $Y$ versus $\sqrt{E_{i}}$ one, although the former is a little lower than the latter by about $\Delta E_{i} \approx 50-150 \mathrm{eV}$ [Figs. 4(b) and 5].

\section{Power spectral density of surface roughness}

To further characterize the roughened surface features, the PSD distribution as well as rms roughness of feature surfaces is important, ${ }^{9,22,23,45}$ because the rms surface roughness reflects only the vertical extent of the feature, while the PSD gives the information on both its vertical and lateral (or spatial) extent. In the PSD analysis, the two-dimensional (2D) PSD function is calculated from the 2D discrete Fourier transform of the 2D surface height or depth distribution; ${ }^{58,59}$ the one-dimensional PSD function is then obtained by angularly averaging the 2D-PSD, retaining a $2 \mathrm{D}$ function with a unit of fourth power to the length.

Figure 10 shows the time evolution of the angularly averaged PSD distribution $P(k)$ for three different ion incident energies of $E_{i} \approx 120,310$, and $470 \mathrm{eV}$, obtained from the PSD analysis of AFM images of the Si surfaces etched in ICP $\mathrm{Cl}_{2}$ plasmas ( $t_{\text {etch }}=1-20 \mathrm{~min}$ ) as shown in Figs. 3 and 8. Also shown is the PSD distribution of wet-cleaned $\mathrm{Si}$ surfaces prior to etching $\left(t_{\mathrm{etch}}=0\right)$ for reference. These $P(k)$ evolutions correspond to the two modes of surface roughening as mentioned above with respect to Figs. 4, 5, and 7. The length scale concerned is the side length $W_{A}=1 \mu \mathrm{m}$ of the AFM scan area with the resolution of 256 pixels (a pixel size $L_{A}=3.9 \mathrm{~nm}$ ), which corresponds to the spatial frequency ranging from $k=0.001$ to $0.128 \mathrm{~nm}^{-1}$ in the reciprocal space. It is generally appreciated that for the PSD curve, the parameters of interest are $e^{9,22,23,45}$ the plateau height value $w_{0}$ at low $k$ related to the vertical height or depth of roughened surface features $\left[P(k) \approx w_{0}\right.$ at $\left.k<k_{0}\right]$, and the correlation length $\xi_{0}\left(=1 / k_{0}\right)$ that defines the lateral size of the roughness; moreover, the curve at high $k$ gives a fractal or selfaffine nature of the roughness $\left[P(k) \approx K / k^{\eta}\right.$ at $\left.k>k_{0}\right],{ }^{59-63}$ where $K$ is the spectral strength, the exponent $\eta$ is a constant linked to the fractal dimension of the surface, and $\xi_{0}$ defines the upper limit of the scale where the surface exhibits the self-affine nature.
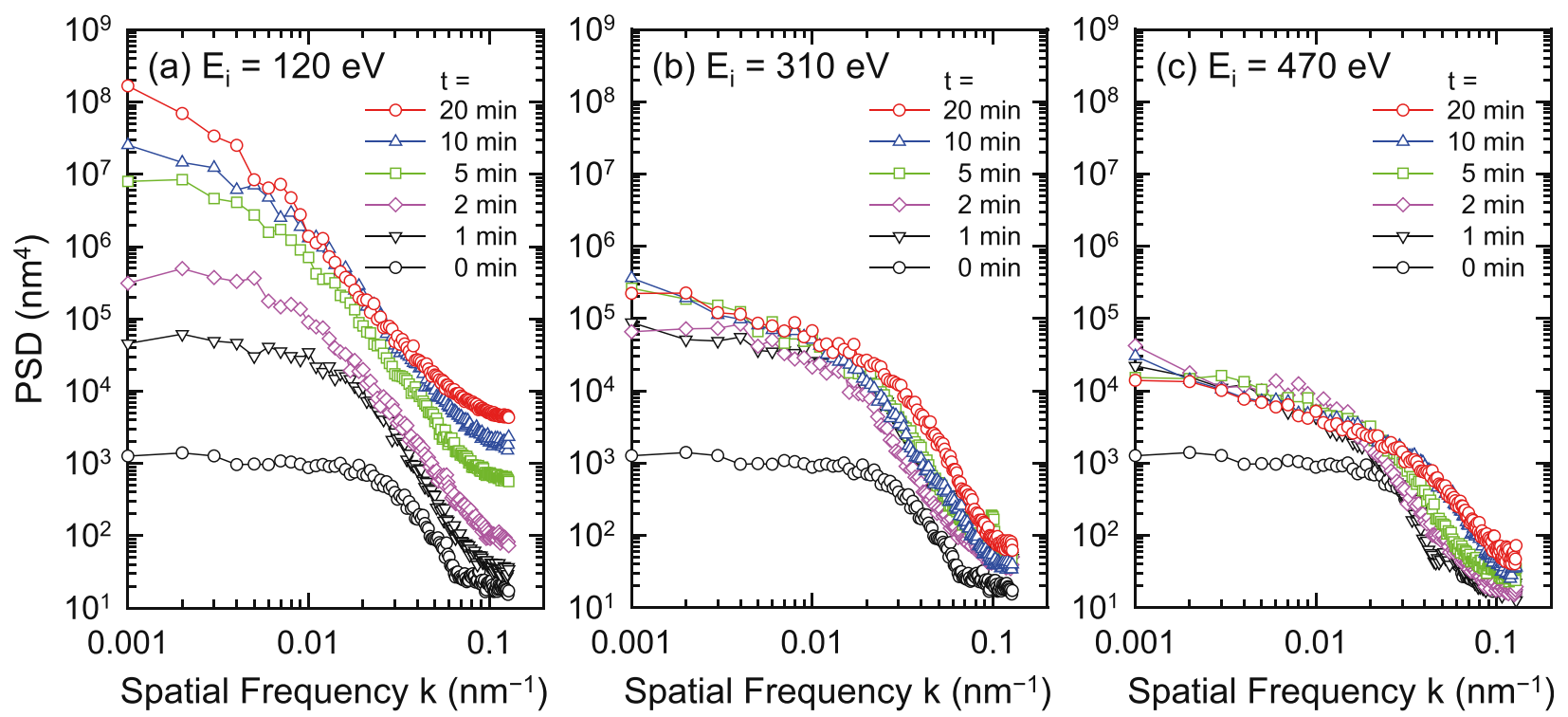

FIG. 10. Time evolution of the angularly averaged PSD distribution $P(k)$ for three different ion incident energies of $E_{i} \approx$ (a) 120 , (b) 310 , and (c) $470 \mathrm{eV}$, obtained from the PSD analysis of AFM images of the Si surfaces etched in $\mathrm{ICP} \mathrm{Cl}_{2}$ plasmas $\left(t_{\mathrm{etch}}=1-20 \mathrm{~min}\right)$ as shown in Figs. 3 and 8 . Also shown is the PSD distribution of wet-cleaned Si surfaces prior to etching $\left(t_{\text {etch }}=0\right)$ for reference. 
The PSD analysis indicates that at $E_{i} \approx 120 \mathrm{eV}$ (roughening mode), the plateau value $w_{0}$ and correlation length $\xi_{0}$ increase monotonically with time during etching, corresponding to the increase in both vertical and lateral roughness; in particular, the correlation length as well as the rms surface roughness increases almost linearly with time (from $\xi_{0} \approx 50 \mathrm{~nm}$ at $t=0$ to $\approx 200 \mathrm{~nm}$ at $t=10 \mathrm{~min}$ ), as has been observed in several plasma etching experiments of $\mathrm{Si}$ in $\mathrm{SF}_{6}$ (Refs. 9 and 20) and $\mathrm{CF}_{4} / \mathrm{O}_{2}{ }^{14}$ Note that the $w_{0}$ and $\xi_{0}$ tend to become unclear at $t>10 \mathrm{~min}$, probably because the lateral size of the increased roughness tends to exceed the limit length scale $W_{A}=1 \mu \mathrm{m}$ or its reciprocal $k=0.001 \mathrm{~nm}^{-1}$ of the present AFM scan area. Moreover, the $P(k)$ tail at high frequencies $\left(k>k_{0}=1 / \xi_{0}\right)$ exhibits a slight increase in spectral strength $K$ during etching, when the fractal-linked constant remains almost unchanged at $\eta \approx 3$ (marginal fractal). ${ }^{59}$ Similar evolution of $P(k)$ was observed for different ion energies $E_{i}<E_{p}(\approx 250 \mathrm{eV})$, where for a given $t_{\text {etch }}$, the $w_{0}$, $\xi_{0}$, and $K$ values are increased at increased $E_{i}$, while that of $\eta$ remains almost the same. These imply that in the roughening mode, the increase in surface roughness with time during etching arises primarily from an increase in height/depth of low-frequency (or long-wavelength) components, without a significant change in height/depth of high-frequency (or short-wavelength) ones; in effect, the frequency independent regime at low frequencies is attributed to the evolution of hole-like microstructures, evident visually in SEM images of Fig. 9, which are randomly situated and separated by roughly similar intervals, and which obviously increase in lateral size as well as vertical depth with time during etching. On the other hand, the high frequency self-affine regime is attributed to the low-level, stochastic roughening originating intrinsically from the temporal as well as spatial uniformity of the incident flux and angle of ions and/or neutral reactants on surfaces at microscale. ${ }^{13-16,21,24,45}$

In contrast, the evolutions of $P(k)$ at $E_{i} \approx 310$ and $470 \mathrm{eV}$ (smoothing mode) exhibit markedly different behaviors: the $P(k)$ curve remains almost unchanged over time during etching $(t>1 \mathrm{~min})$, after some change at the initial stage of etching $(t<1 \mathrm{~min})$; concretely, the correlation length $\left(\xi_{0} \approx 50 \mathrm{~nm}\right)$ remains almost the same as that of wet-cleaned surfaces prior to etching $\left(t_{\text {etch }}=0\right)$, and the $P(k)$ tail at high frequencies $\left(k>k_{0}=1 / \xi_{0} \approx 0.02 \mathrm{~nm}^{-1}\right)$ remains similar to that of the wet-cleaned surfaces with a fractal-linked constant $\eta \approx 3$, although the $P(k)$ tail exhibits a little increase in spectral strength $K$ with time during etching. On the other hand, at low frequencies $\left(k<k_{0}\right)$, the $P(k)$ magnitude increases slightly at the initial stage of etching, and it remains almost unchanged thereafter; then, the plateau value $w_{0}$ becomes unclear, and the $P(k)$ curve exhibits a second self-affine surface, remaining almost unchanged with a second spectral strength $K^{\prime}$ and fractal constant $\eta^{\prime} \approx 1$ (extreme fractal). ${ }^{59}$ Such multiple self-affine surfaces have been observed in thin film deposition experiments, being correlated to the formation and evolution of film microstructures. ${ }^{60-63}$ Similar evolution of $P(k)$ was observed for different ion energies $E_{i}>E_{p}$ $(\approx 250 \mathrm{eV})$, where for a given $t_{\text {etch }}$, the $K$ and $K^{\prime}$ values are decreased at increased $E_{i}$, while those of $\xi_{0}, \eta$, and $\eta^{\prime}$ remain almost the same, as can be seen from a comparison between the $P(k)$ evolutions at $E_{i} \approx 310$ and $470 \mathrm{eV}$. These imply that in the smoothing mode, only the low-level, stochastic roughening occurs on surfaces after some increase in roughness at the initial stage of etching, where the two self-affine fractal regimes at low and high frequencies are attributed to the stochastic roughening associated with the incoming flux of ionic species of different natures for surface reaction kinetics, as will be discussed below in Secs. III D and III E.

\section{Etch products and byproducts}

\section{Optical emission spectroscopy}

Figure 11 shows typical OES spectra in the wavelength range 200-900 nm during ICP plasma etching of Si in $\mathrm{Cl}_{2}$, observed for four different ion incident energies of $E_{i} \approx 22-470 \mathrm{eV}$ under the same conditions as in Fig. 2. Also shown is an OES spectrum from the $\mathrm{Cl}_{2}$ plasma in the absence of etching ( $P_{\mathrm{rf}}=0 \mathrm{~W}$ or $E_{i} \approx 13 \mathrm{eV}$ without breakthrough step) for reference. In the uv range, atomic lines and molecular bands originating from etch products and/or byproducts become more significant at increased $P_{\mathrm{rf}}$ or $E_{i}$, corresponding to the increase in etch rate as shown in Fig. 4(a) and the resultant increase in their concentration in the plasma: ${ }^{64-67} \mathrm{SiCl}$ bands are identified at 280.7 and $282.3 \mathrm{~nm},{ }^{68-70}$ a broad band of $\mathrm{SiCl}_{2}$ around $330 \mathrm{~nm},{ }^{71-75}$ and another broad one of $\mathrm{SiCl}_{2},{ }^{72-74} \mathrm{SiCl}_{3},{ }^{66,67}$ or $\mathrm{Si}_{2}$ (Refs. 68, 69, 75, and 76) around $390 \mathrm{~nm}$, together with $\mathrm{Si}$

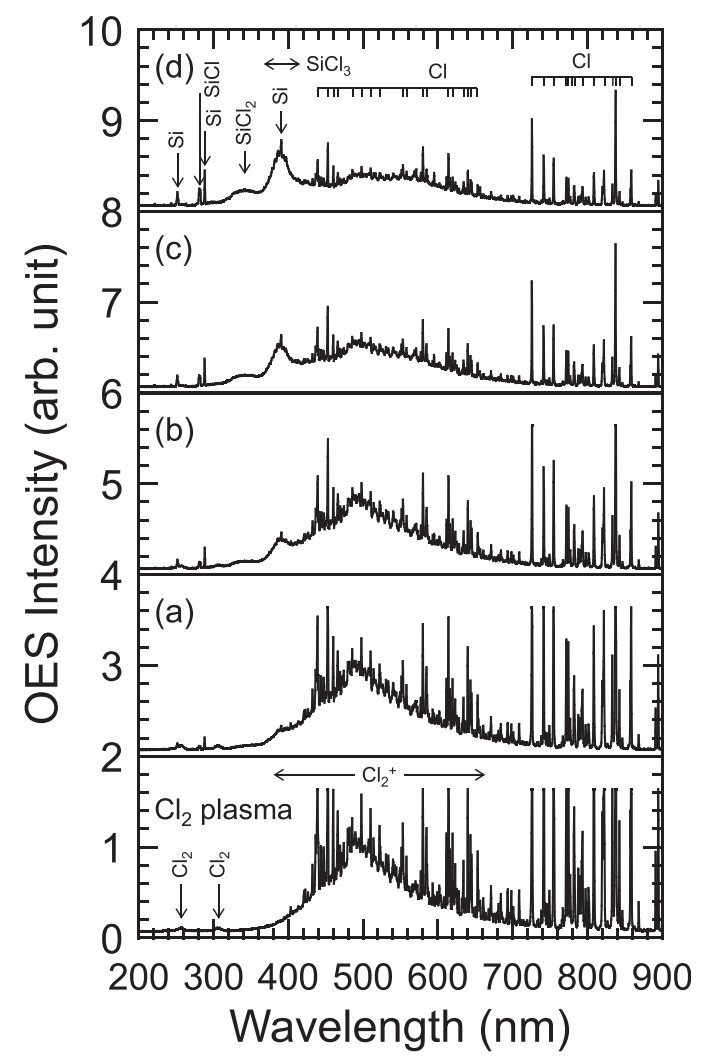

FIG. 11. Typical OES spectra in the wavelength range 200-900 nm during ICP plasma etching of $\mathrm{Si}$ in $\mathrm{Cl}_{2}$, observed for four different ion incident energies of $E_{i} \approx$ (a) 22 , (b) 120 , (c) 220 , and (d) $470 \mathrm{eV}$ under the same conditions as in Fig. 2. Also shown is an OES spectrum from the $\mathrm{Cl}_{2}$ plasma in the absence of etching ( $P_{\mathrm{rf}}=0 \mathrm{~W}$ or $E_{i} \approx 13 \mathrm{eV}$ without breakthrough step) for reference. 


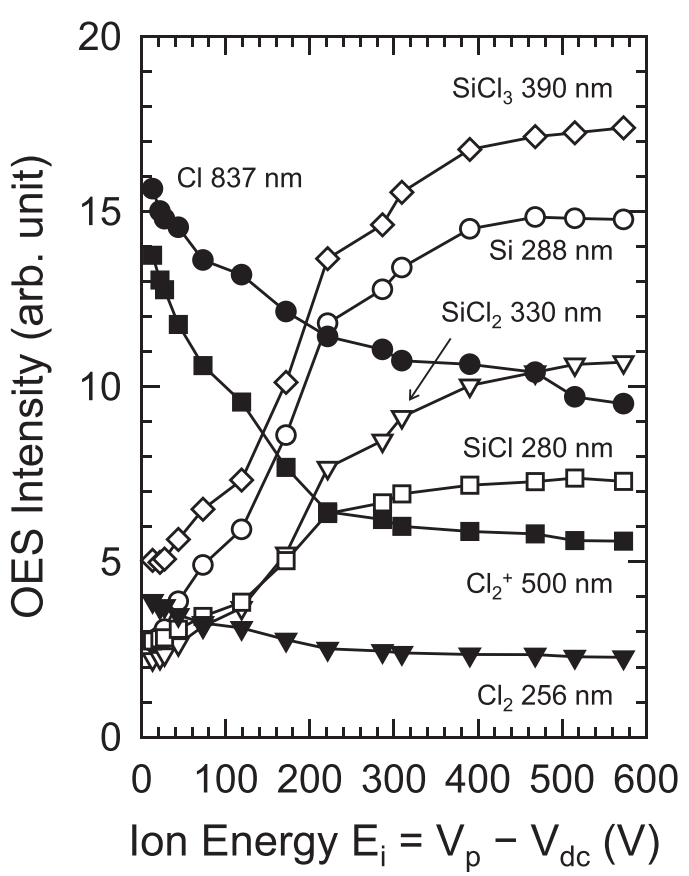

FIG. 12. OES intensities of seven species $\operatorname{SiCl}_{x}(x=0-3), \mathrm{Cl}_{x}(x=1,2)$ and $\mathrm{Cl}_{2}{ }^{+}$as a function of ion incident energy in the range $E_{i} \approx 13-570 \mathrm{eV}$, measured during ICP plasma etching of $\mathrm{Si}$ in $\mathrm{Cl}_{2}$ under the same conditions as in Fig. 2. The wavelengths and transitions for the atomic and molecular emissions concerned are listed in Table I.

lines at $251.6,288.1$, and $390.5 \mathrm{~nm} .{ }^{77}$ It is noted here that in this paper, etch byproducts mean $\mathrm{Si}$-containing species (ions and neutrals) formed through ionization, dissociation, and/or reaction of etch products (primary products) after being desorbed from substrate surfaces during etching. In contrast, spectral lines and bands originating from feed gases become less significant at increased $P_{\text {rf }}$ or $E_{i}$, corresponding to reduced partial pressures or concentrations of feed gases under the operating conditions of constant pressure $P_{0}$ : weak $\mathrm{Cl}_{2}$ emission bands identified at 256.6 and $306.3 \mathrm{~nm}$ in the uv, ${ }^{65,68,69,78-80}$ and broad, prominent bands of $\mathrm{Cl}_{2}{ }^{+}$extending from 380 to $660 \mathrm{~nm}$ in the visible, ${ }^{68,69,81-83}$ together with numerous $\mathrm{Cl}$ lines (strong peaks at $438.9,452.6,579.6$, and $614.0 \mathrm{~nm})^{77}$ superimposed thereon, and those in the near-IR range (strong peaks at $725.6,741.4,754.7,837.5$, and $858.5 \mathrm{~nm}) .{ }^{77} \mathrm{The} \mathrm{Cl}^{+}$ion lines in the visible were not identified in the present experiments, owing to relatively low electron temperatures $T_{e}$ as compared with electron cyclotron resonance (ECR) plasmas operated at $P_{0}<1$ mTorr. ${ }^{46,47,64}$

Figure 12 shows the OES intensities of seven species $\mathrm{SiCl}_{x}(x=0-3), \mathrm{Cl}_{x}(x=1,2)$, and $\mathrm{Cl}_{2}{ }^{+}$as a function of ion incident energy in the range $E_{i} \approx 13-570 \mathrm{eV}$, measured during ICP plasma etching of $\mathrm{Si}$ in $\mathrm{Cl}_{2}$ under the same conditions as in Fig. 2. The wavelengths and transitions for the atomic and molecular emissions concerned are listed in Table I. Note that the actinometry ${ }^{84}$ with a small amount of inert gas (actinometer) added to feed gases was not employed in these measurements, because at increased $P_{\mathrm{rf}}$ or $E_{i}$, the amount of Si etched per minute is increased and comparable with that of feed gases (as mentioned earlier with respect to Fig. 4); thus, the partial pressure of feed gases and so the concentration of the actinometer in the plasma is decreased at increased $P_{\mathrm{rf}}$ or $E_{i}$, where the optical emission intensity ratio of the target species to the actinometer would not be related to the species concentration therein. In the present experiments, the raw emission intensity was assumed to represent qualitatively the trend in species concentration in the plasma, because no significant change in electron density and temperature occurred over the operating range investigated (as in Fig. 2). The results indicate that as the bias power $P_{\mathrm{rf}}$ or ion energy $E_{i}$ is increased, the intensities or concentrations of etch product/byproduct neutrals $\mathrm{SiCl}_{x}$ $(x=0-3)$ increase and then tend to be saturated, while those of feed gas neutrals $\mathrm{Cl}_{x}(x=1,2)$ and ions $\mathrm{Cl}_{2}{ }^{+}$decrease monotonically.

Similar behavior of the OES intensities was observed for different gas flow rates $F_{0}$; in practice, at a given $P_{\text {rf }}$ or

TABLE I. Wavelengths and transitions for atomic and molecular emissions concerned in this study of $\mathrm{Cl}_{2}$ plasma etching of Si.

\begin{tabular}{|c|c|c|c|c|}
\hline Species & Wavelength (nm) & Transition lower-upper & Energy $(\mathrm{eV})$ lower-upper & References \\
\hline $\mathrm{Si}$ & 288.1 & $3 p^{2}\left({ }^{1} D_{2}\right)-4 s\left({ }^{1} P^{\mathrm{o}}{ }_{1}\right)$ & $0.78-5.08^{\mathrm{a}}$ & 77 \\
\hline $\mathrm{SiCl}$ & 280.7 & $X^{2} \Pi_{r}\left(v^{\prime \prime}=0\right)-B^{\prime 2} \Delta\left(v^{\prime}=2\right)$ & $0-4.41^{\mathrm{b}}$ & $68-70$ \\
\hline $\mathrm{SiCl}_{2}$ & 330 & $\tilde{X}^{1} A_{1}\left(v^{\prime \prime}=0\right)-\tilde{A}^{1} B_{1}\left(v^{\prime}=0\right)$ & $0-3.76^{\mathrm{c}}$ & $71-75$ \\
\hline $\mathrm{SiCl}_{2}$ & ) & $\tilde{X}^{1} A_{1}-\tilde{A}^{1} B_{1}$ & & $72-74$ \\
\hline $\mathrm{SiCl}_{3}$ & 390 & & & 66,67 \\
\hline $\mathrm{Si}_{2}$ & J & $X^{3} \Sigma_{g}^{-}\left(v^{\prime \prime}=0\right)-H^{3} \Sigma_{u}^{-}\left(v^{\prime}=5\right)$ & $0-3.17^{\mathrm{d}}$ & $68,69,75,76$ \\
\hline $\mathrm{Cl}$ & 837.5 & $4 s\left({ }^{4} P_{5 / 2}\right)-4 p\left({ }^{4} D^{\mathrm{o}}{ }_{7 / 2}\right)$ & $8.92-10.40^{\mathrm{a}}$ & 77 \\
\hline $\mathrm{Cl}_{2}$ & 256.6 & $\begin{array}{c}1\left({ }^{3} \Pi_{u}\right)\left[A^{\prime} 2_{u}\left({ }^{3} \Pi\right)\right]\left(v^{\prime \prime}=5\right) \\
-2\left({ }^{3} \Pi_{g}\right)\left[D^{\prime} 2_{g}\left({ }^{3} P_{2}\right)\right]\left(v^{\prime}=0\right)\end{array}$ & $2.27-7.10^{\mathrm{e}}$ & $65,68,69,78-80$ \\
\hline $\mathrm{Cl}_{2}^{+}$ & 500.7 & $\begin{array}{c}{ }^{2} \Pi_{3 / 2}\left[\tilde{X}^{2} \Pi_{g}\right]\left(v^{\prime \prime}=4\right) \\
-{ }^{2} \Pi_{3 / 2}\left[\tilde{A}^{2} \Pi_{g}\right]\left(v^{\prime}=0\right)\end{array}$ & $0-2.48^{\mathrm{f}}$ & $68,69,81-83$ \\
\hline
\end{tabular}

${ }^{\mathrm{a}}$ Reference 77.

${ }^{\mathrm{b}}$ Reference 69.

${ }^{\mathrm{c}}$ Reference 73 .

${ }^{\mathrm{d}}$ Reference 76.

${ }^{\mathrm{e}}$ References 79 and 80.

${ }^{\mathrm{f}}$ References 69 and 81. 
$E_{i}$, the intensities or concentrations of etch product/byproduct species were found to be decreased for increased $F_{0}$, while those of feed gas species were increased, as partly shown in Fig. 13 for atomic $\mathrm{Si}$ and $\mathrm{Cl}$. The former reflects shortened residence times and thus the reduced concentrations of products/byproducts in the plasma, and the latter reflects the consequently increased partial pressures or concentrations of feed gases under the operating conditions of constant pressure $P_{0}$. It is further noted that the $F_{0}$ as well as $P_{\mathrm{rf}}$ or $E_{i}$ dependence of the $\mathrm{Cl}$ emission intensity $I_{\mathrm{Cl}}$ was very similar to that of the $\mathrm{Cl}_{2}$ one $I_{\mathrm{Cl}_{2}}$, implying that the $\mathrm{Cl}$ intensity represents the trend in feed $\mathrm{Cl}_{2}$ gas density in the plasma.

\section{Infrared absorption spectroscopy}

Figure 14 shows the peak absorbance of $\mathrm{SiCl}_{4}$ at around $620 \mathrm{~cm}^{-1}\left(\nu_{3} \text { fundamental vibrational band }\right)^{46,47,85}$ as a function of ion incident energy in the range $E_{i} \approx 13-570 \mathrm{eV}$, measured by FTIR-TAS during ICP plasma etching of Si in $\mathrm{Cl}_{2}$ for four different gas flow rates of $F_{0}=5-50 \mathrm{sccm}$ under otherwise the same conditions as in Fig. 2. The calibration was made by filling the ICP reactor chamber with pure $\mathrm{SiCl}_{4}$ gases at different pressures in the range $P_{0}=0.05-50 \mathrm{mTorr}$ without discharge, to derive the absolute concentration of etch product/byproduct neutrals $\mathrm{SiCl}_{4}$ in the plasma during etching. ${ }^{46,47}$ The results indicate that for any $F_{0}$, the absorbance of the $620-\mathrm{cm}^{-1} \mathrm{SiCl}_{4}$ band and thus the concentration of $\mathrm{SiCl}_{4}$ increases and then tends to be saturated with increasing $P_{\mathrm{rf}}$ or $E_{i}$, corresponding to the increase in etch rate; moreover, at a given $P_{\text {rf }}$ or $E_{i}$, the absorbance and thus the concentration of $\mathrm{SiCl}_{4}$ is decreased for increased flow rates $F_{0}$, corresponding to shortened residence times of it.

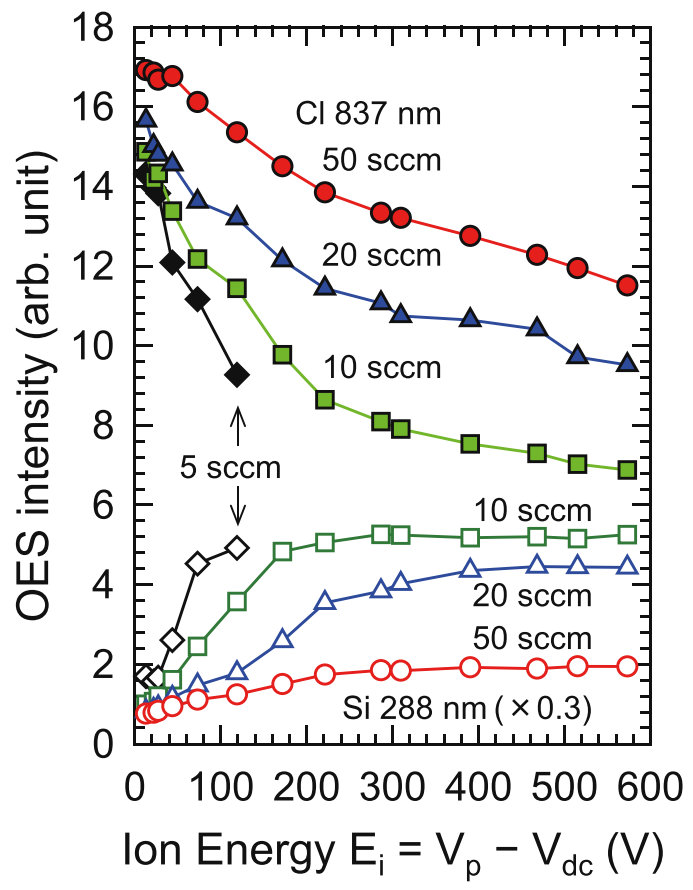

FIG. 13. OES intensities of two atomic lines of $\mathrm{Si}$ and $\mathrm{Cl}$ as a function of ion incident energy in the range $E_{i} \approx 13-570 \mathrm{eV}$, measured during ICP plasma etching of $\mathrm{Si}$ in $\mathrm{Cl}_{2}$ for four different gas flow rates of $F_{0}=5-50 \mathrm{sccm}$ under otherwise the same conditions as in Fig. 2.

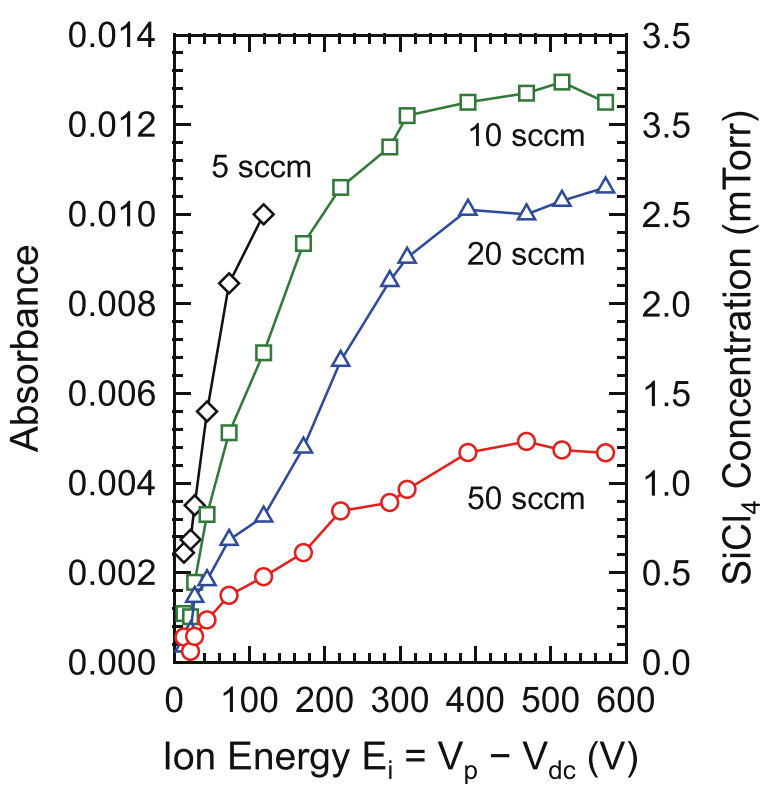

FIG. 14. Peak absorbance of $\mathrm{SiCl}_{4}$ at around $620 \mathrm{~cm}^{-1}$ as a function of ion incident energy in the range $E_{i} \approx 13-570 \mathrm{eV}$, measured by FTIR-TAS during ICP plasma etching of $\mathrm{Si}$ in $\mathrm{Cl}_{2}$ for four different gas flow rates of $F_{0}=5-50 \mathrm{sccm}$ under otherwise the same conditions as in Fig. 2. The calibration was made by filling the ICP reactor chamber with pure $\mathrm{SiCl}_{4}$ gases at different pressures in the range $P_{0}=0.05-50$ mTorr without discharge to derive the absolute concentration of etch product/byproduct neutrals $\mathrm{SiCl}_{4}$ in the plasma during etching.

These are consistent with the behavior $\left(E_{i}\right.$ and $F_{0}$ dependences) of the OES intensities of $\operatorname{SiCl}_{x}(x=0-3)$ as shown above in Figs. 12 and 13. The concentration of $\mathrm{SiCl}_{4}$ in the plasma during etching is estimated to be more than $10 \%$ of the feed $\mathrm{Cl}_{2}$ gas density, at increased $P_{\mathrm{rf}}$ or $E_{i}$ and for decreased $F_{0}$; e.g., $\left[\mathrm{SiCl}_{4}\right] /\left[\mathrm{Cl}_{2}\right]_{0}>0.1$ (or $\left[\mathrm{SiCl}_{4}\right]>6.4 \times 10^{13} \mathrm{~cm}^{-3}$ at $P_{0}=20 \mathrm{mTorr}$ ), at $E_{i}>250 \mathrm{eV}$ for $F_{0}=20 \mathrm{sccm}$ and at $E_{i}>150 \mathrm{eV}$ for $F_{0}=10 \mathrm{sccm}$, where $\left[\mathrm{Cl}_{2}\right]_{0}$ denotes the $\mathrm{Cl}_{2}$ density under plasma-off conditions. The $\mathrm{SiCl}_{4}$ partial pressure or concentration presently measured is in agreement with previous QMS measurements during plasma etching of $\mathrm{Si}$ in $\mathrm{Cl}_{2}{ }^{86,87}$

It is further noted that in a rough approximation, taking account of the recent modeling simulations of ICP plasma etching of $\mathrm{Si}$ in $\mathrm{Cl}_{2}$ that the most common etch product/ byproduct neutrals in the plasma are $\operatorname{SiCl}_{x}(x=2-4)$ with comparable densities ${ }^{88}$ the total amount of them is estimated to be $\sum_{x}\left[\mathrm{SiCl}_{x}\right] /\left[\mathrm{Cl}_{2}\right]_{0}>0.3$ under the above conditions. This is consistent with the OES data for feed gas neutrals as shown in Figs. 12 and 13: $I_{\mathrm{Cl}} /\left(I_{\mathrm{Cl}}\right)_{0} \approx I_{\mathrm{Cl}_{2}} /\left(I_{\mathrm{Cl}_{2}}\right)_{0} \approx\left[\mathrm{Cl}_{2}\right] /$ $\left[\mathrm{Cl}_{2}\right]_{0}<0.7$ under these conditions, assuming low degree of dissociation of $\mathrm{Cl}_{2}$ in the plasma. Here, $\left(I_{\mathrm{Cl}}\right)_{0}$ and $\left(I_{\mathrm{Cl}_{2}}\right)_{0}$ denote the $\mathrm{Cl}$ and $\mathrm{Cl}_{2}$ emission intensities in the absence of etching ( $P_{\mathrm{rf}}=0 \mathrm{~W}$ or $E_{i} \approx 13 \mathrm{eV}$ without breakthrough step).

\section{Mass spectrometry}

Figure 15 shows typical QMS spectra in the mass range $m / e=1-200 \mathrm{amu}$ during ICP plasma etching of $\mathrm{Si}$ in $\mathrm{Cl}_{2}$, measured with the ionizer (using $70-\mathrm{eV}$ electrons) switched on and off, for an ion incident energy of $E_{i} \approx 120 \mathrm{eV}$ under the same conditions as in Fig. 2. In the spectra, the signals of 


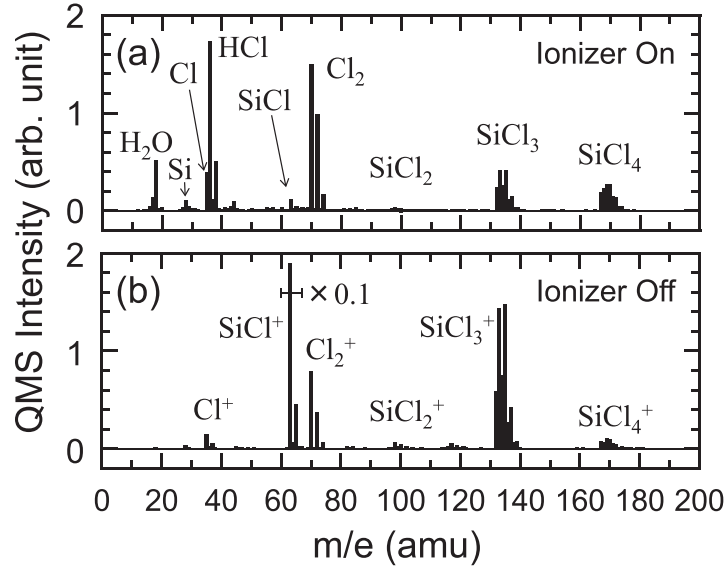

FIG. 15. Typical QMS spectra in the mass range $m / e=1-200 \mathrm{amu}$ during ICP plasma etching of $\mathrm{Si}$ in $\mathrm{Cl}_{2}$, measured with the ionizer (using 70-eV electrons) switched (a) on and (b) off, for an ion incident energy of $E_{i} \approx$ $120 \mathrm{eV}$ under the same conditions as in Fig. 2. In the spectra, the signals of $\mathrm{Si}$ - and $\mathrm{Cl}$-containing species consist of multiple peaks originating from naturally abundant isotopes of $\mathrm{Si}\left({ }^{28} \mathrm{Si},{ }^{29} \mathrm{Si},{ }^{30} \mathrm{Si}\right)$ and $\mathrm{Cl}\left({ }^{35} \mathrm{Cl},{ }^{37} \mathrm{Cl}\right)$.

$\mathrm{Si}$ - and Cl-containing species consist of multiple peaks originating from naturally abundant isotopes of $\mathrm{Si}\left({ }^{28} \mathrm{Si},{ }^{29} \mathrm{Si}\right.$, $\left.{ }^{30} \mathrm{Si}\right)$ and $\mathrm{Cl}\left({ }^{35} \mathrm{Cl},{ }^{37} \mathrm{Cl}\right)$; in addition, the signal intensities of Si-containing species were found to be lowered by several orders of magnitude in the absence of etching $\left(P_{\mathrm{rf}}=0 \mathrm{~W}\right.$ or $E_{i} \approx 13 \mathrm{eV}$ without breakthrough), implying that they originate primarily from etch products desorbed from substrate surfaces during etching. The ion mass spectrum with the ionizer off indicates that the predominant ions are ionized etch products/byproducts, $\mathrm{SiCl}^{+}$and $\mathrm{SiCl}_{3}{ }^{+}$followed by $\mathrm{SiCl}_{4}{ }^{+}$, which are more abundant than the dominant feed gas ions $\mathrm{Cl}_{2}{ }^{+}$, as has been observed and/or concluded in a few QMS measurements during $\mathrm{Cl}_{2}$ and $\mathrm{Cl}_{2}$-containing plasma etching of $\mathrm{Si}^{89-91}$ This essential feature for the predominant ionic species during etching $\left(\mathrm{SiCl}_{x}^{+}\right.$, and not $\left.\mathrm{Cl}_{x}^{+}\right)$has been different from most modeling predictions, ${ }^{92-94}$ while has lastly been predicted by the recent modeling simulations. ${ }^{88}$ The neutral mass spectrum with the ionizer on exhibits several peaks of impurity species $\mathrm{O}, \mathrm{OH}, \mathrm{H}_{2} \mathrm{O}$, and $\mathrm{HCl}(\mathrm{m} / e=16$, 17,18 , and 36/38), in addition to peaks of etch product/ byproduct $\mathrm{SiCl}_{x}(x=0-4)$ and feed gas $\mathrm{Cl}_{x}(x=1,2)$ species. In practice, $\mathrm{O}, \mathrm{OH}$, and $\mathrm{H}_{2} \mathrm{O}$ were detected in the neutral spectrum, even when the ICP reactor chamber was pumped down to a base pressure, and the $\mathrm{HCl}$ signal was increased under plasma-on conditions, implying that these impurities detected arise probably from $\mathrm{H}_{2} \mathrm{O}$ adsorbed on the QMS chamber walls.

It is further noted that weak signals were detected around $m / e=44(\mathrm{SiO})^{89}$ and $247\left(\mathrm{Si}_{2} \mathrm{OCl}_{5}\right)^{64,87}$ in both the ion and neutral mass spectra, and around $m / e=310\left(\mathrm{Si}_{3} \mathrm{OCl}_{6}\right)$ and $361\left(\mathrm{Si}_{3} \mathrm{O}_{2} \mathrm{Cl}_{7}\right)$ in the ion spectrum, while no significant signal was detected around $m / e=231,266$, and $301\left(\mathrm{Si}_{2} \mathrm{Cl}_{x}\right.$; $x=5,6$, and 7$)^{64,87}$ in both spectra. These O-containing byproducts detected (silicon oxides and oxychlorides) arise probably from reactions of etch products/byproducts (silicon chlorides) with $\mathrm{O}$ impurities sputtered from the dielectric window at the top of the ICP reactor chamber; in practice, a small amount of oxygen was also assumed to occur therein under plasma-on conditions, in view of the FTIR-TAS spectra that exhibited a weal absorption of $\mathrm{SiO}$ at around $1100 \mathrm{~cm}^{-1}, 46,47,69,85,95$ although the $\mathrm{O}$ line emissions (e.g., peaks at 777.1-777.5 and $844.6 \mathrm{~nm})^{77}$ were not detected in the OES observations. In the present experiments, only the ion mass spectrum was analyzed to investigate the composition of the ion flux entering the mass spectrometer and similarly incident on substrate surfaces from the plasma, because the neutral spectrum requires careful attention and differential pumping systems to gain quantitative information on incoming neutral fluxes. ${ }^{86,87,96}$

Figure 16 shows the integrated QMS intensities of seven ionic species $\mathrm{SiCl}_{x}{ }^{+}(x=0-4)$ and $\mathrm{Cl}_{x}^{+}(x=1,2)$ as a function of ion incident energy in the range $E_{i} \approx 13-570 \mathrm{eV}$, measured with the ionizer off during ICP plasma etching of $\mathrm{Si}$ in $\mathrm{Cl}_{2}$ under the same conditions as in Fig. 2. Note that in these measurements, the raw signal intensity (counts/s) of ionic species was corrected, taking into account the transmission and detection efficiency of the spectrometer depending on ion mass; ${ }^{89}$ then, the corrected signal intensities of isotopic components of the respective ions were integrated to estimate the incoming flux of the ionic species concerned. The results indicate that as the bias power $P_{\mathrm{rf}}$ or ion energy $E_{i}$ is increased, the intensities or fluxes of ionized etch products/byproducts $\mathrm{SiCl}_{x}^{+}(x=0,1,2,4)$ increase and then tend to be saturated, corresponding to the increase in etch rate, while $\mathrm{SiCl}_{3}{ }^{+}$exhibits a little different behavior that its flux increases, peaks, and then decreases. This is ascribed presumably to etch product species desorbed depending on $E_{i}$;

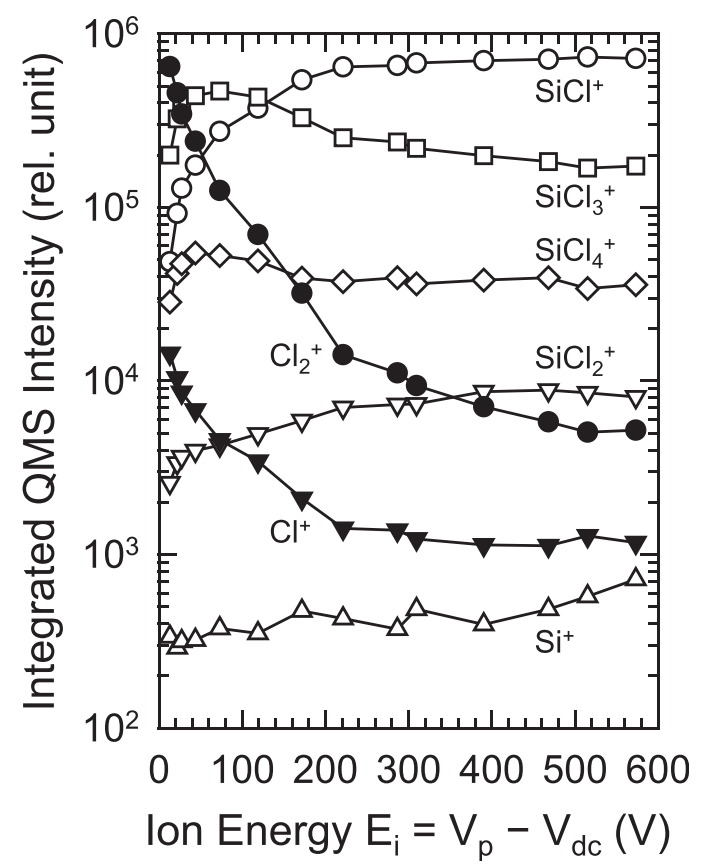

FIG. 16. Integrated QMS intensities of seven ionic species $\mathrm{SiCl}_{x}^{+}(x=0-4)$ and $\mathrm{Cl}_{x}^{+}(x=1,2)$ as a function of ion incident energy in the range $E_{i} \approx$ 13-570 eV, measured with the ionizer switched off during ICP plasma etching of $\mathrm{Si}$ in $\mathrm{Cl}_{2}$ under the same conditions as in Fig. 2. In these measurements, the raw signal intensity (counts/s) of ionic species was corrected, taking into account the transmission and detection efficiency of the spectrometer depending on ion mass; then, the corrected signal intensities of isotopic components of the respective ions were integrated to estimate the incoming flux of the ionic species concerned. 
in practice, the primary etch products desorbed from substrate surfaces during $\mathrm{Si}$ etching in $\mathrm{Cl}_{2}$ plasmas are generally assumed to be saturated $\mathrm{SiCl}_{4}$ at low $E_{i}<100 \mathrm{eV}$, while unsaturated $\operatorname{SiCl}_{x}(x=1,2)$ at higher $E_{i}{ }^{97-100}$ Under plasma electron-impact ionization in the energy range concerned $\left(E_{e}<\mathrm{a}\right.$ few tens of eV), we may consider that $\mathrm{SiCl}_{4}$ molecules predominantly fragment into $\mathrm{SiCl}_{3}{ }^{+}$, followed by $\mathrm{SiCl}_{4}{ }^{+}$and $\mathrm{SiCl}^{+},{ }^{101-103}$ and that $\mathrm{SiCl}_{2}$ and $\mathrm{SiCl}$ yield predominantly $\mathrm{SiCl}_{2}{ }^{+}$and $\mathrm{SiCl}^{+}$, respectively. ${ }^{104,105}$ These situations appear to lead to the present QMS measurements that $\mathrm{SiCl}_{3}{ }^{+}$is the predominant Si-containing ion flux at low $E_{i}<120 \mathrm{eV}$, followed by $\mathrm{SiCl}^{+}$and $\mathrm{SiCl}_{4}^{+}$, while $\mathrm{SiCl}^{+}$is that at higher $E_{i}$, followed by $\mathrm{SiCl}_{3}{ }^{+}$and $\mathrm{SiCl}_{4}{ }^{+}$.

On the other hand, the intensities or fluxes of feed gas ions $\mathrm{Cl}_{x}{ }^{+}(x=1,2)$ decrease monotonically with increasing $E_{i}$, corresponding to the increase in etch rate and the consequent reduced partial pressures or concentrations of feed gases under the operating conditions of constant pressure $P_{0}$; in practice, $\mathrm{Cl}_{2}{ }^{+}$is much more abundant than $\mathrm{Cl}^{+}$, probably because $\mathrm{Cl}_{2}$ molecules yield predominantly $\mathrm{Cl}_{2}{ }^{+}$under electron-impact ionization in the plasma concerned. ${ }^{106,107}$ As a result, the predominant ion fluxes during etching are those of ionized feed gas molecules $\mathrm{Cl}_{2}{ }^{+}$at low $E_{i}<30 \mathrm{eV}$, while those of ionized etch products/byproducts $\mathrm{SiCl}_{3}{ }^{+}$at higher $E_{i}>30 \mathrm{eV}$ and those of $\mathrm{SiCl}^{+}$at further increased $E_{i}>120 \mathrm{eV}$. It should be noted here that at $E_{i} \approx 30 \mathrm{eV}$ for $F_{0}=20 \mathrm{sccm}$ [where the $\mathrm{Si}$ etch rate is $\mathrm{ER} \approx 100 \mathrm{~nm} / \mathrm{min}$ as in Fig. 4(a)], e.g., the respective ion fluxes and concentrations concerned are estimated from the QMS data of Fig. 16 (calibrated by the probe ion saturation current) as $\Gamma\left(\mathrm{SiCl}_{3}{ }^{+}\right) \approx 1.9 \times 10^{15} \mathrm{~cm}^{-2} \mathrm{~s}^{-1}, \Gamma\left(\mathrm{SiCl}^{+}\right)$ $\approx 0.64 \times 10^{15} \mathrm{~cm}^{-2} \mathrm{~s}^{-1}$, and $\Gamma\left(\mathrm{Cl}_{2}{ }^{+}\right) \approx 1.7 \times 10^{15} \mathrm{~cm}^{-2} \mathrm{~s}^{-1}$ (total ion flux $\Gamma_{i}^{0} \approx 5.0 \times 10^{15} \mathrm{~cm}^{-2} \mathrm{~s}^{-1}$ ); $n\left(\mathrm{SiCl}_{3}{ }^{+}\right) \approx 1.1$ $\times 10^{10} \mathrm{~cm}^{-3}, n\left(\mathrm{SiCl}^{+}\right) \approx 0.37 \times 10^{10} \mathrm{~cm}^{-3}$, and $n\left(\mathrm{Cl}_{2}{ }^{+}\right) \approx 1.0$ $\times 10^{10} \mathrm{~cm}^{-3}$ (total ion density $n_{i} \approx 2.9 \times 10^{10} \mathrm{~cm}^{-3}$ ). Thus, it is concluded that under most conditions in these experiments, the fluxes and concentrations of etch product/byproduct ions $\mathrm{SiCl}_{x}{ }^{+}$account for more than half of the total ion flux and density, although their neutrals $\mathrm{SiCl}_{x}$ represent a small fraction of the total species density in the reactor chamber; e.g., under the above conditions, $\left[\mathrm{SiCl}_{4}\right] /\left[\mathrm{Cl}_{2}\right]_{0} \approx 0.019$ and $\sum_{x}\left[\mathrm{SiCl}_{x}\right] /\left[\mathrm{Cl}_{2}\right]_{0}$ $\approx 0.057$, as estimated from the FTIR-TAS data of Fig. 14 . These situations have so far been indicated in only a few QMS measurements ${ }^{89-91}$ and modeling calculations, ${ }^{88}$ being ascribed partly to the difference between the total electron ionization cross sections for $\mathrm{SiCl}_{x}(x=1-4)^{101,104,105,108,109}$ and $\mathrm{Cl}_{2}$ (Refs. 107, 110-112) molecules; in effect, the former is about 2-3 times larger than that the latter.

Similar behavior of the integrated QMS intensities was observed for different gas flow rates $F_{0}$; in practice, at a given $P_{\mathrm{rf}}$ or $E_{i}$, the intensities or fluxes of ionized etch products/byproducts were found to be decreased for increased $F_{0}$, while those of feed gas ions were increased. The $E_{i}$ and $F_{0}$ dependences of the QMS intensities of $\mathrm{SiCl}_{x}^{+}(x=0-4)$ and $\mathrm{Cl}_{x}^{+}(x=1,2)$ ions are consistent with those of the OES intensities of $\mathrm{SiCl}_{x}(x=0-3), \mathrm{Cl}_{x}(x=1,2)$, and $\mathrm{Cl}_{2}{ }^{+}$as shown in Figs. 12 and 13, and also with those of the FTIRTAS absorbance of $\mathrm{SiCl}_{4}$ as in Fig. 14. Figure 17 shows the total integrated QMS intensity of $\mathrm{SiCl}_{3}{ }^{+}, \mathrm{SiCl}_{4}{ }^{+}, \mathrm{Cl}^{+}$, and $\mathrm{Cl}_{2}{ }^{+}$ions and that of $\mathrm{Si}^{+}, \mathrm{SiCl}^{+}$, and $\mathrm{SiCl}_{2}{ }^{+}$ions as a

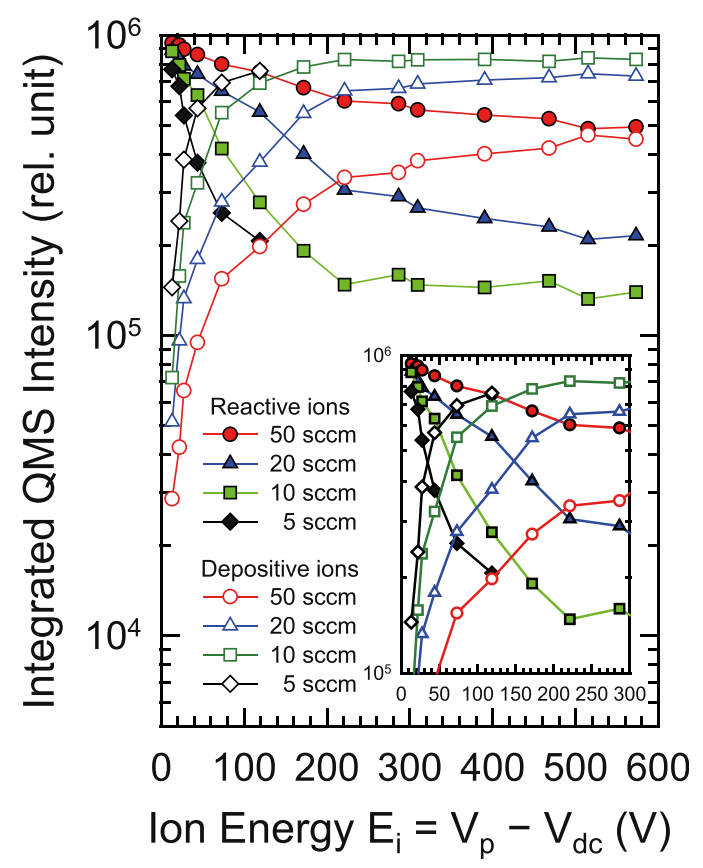

FIG. 17. Total integrated QMS intensity or incoming flux of $\mathrm{SiCl}_{3}{ }^{+}, \mathrm{SiCl}_{4}{ }^{+}$, $\mathrm{Cl}^{+}$, and $\mathrm{Cl}_{2}{ }^{+}$ions and that of $\mathrm{Si}^{+}, \mathrm{SiCl}^{+}$, and $\mathrm{SiCl}_{2}{ }^{+}$ions as a function of ion incident energy in the range $E_{i} \approx 13-570 \mathrm{eV}$, measured with the ionizer off during ICP plasma etching of $\mathrm{Si}$ in $\mathrm{Cl}_{2}$ for four different gas flow rates of $F_{0}=5-50 \mathrm{sccm}$ under otherwise the same conditions as in Fig. 2. These data are those obtained by summing the respective ion intensities as shown in the preceding Fig. 16, and inset is an enlarged view of the figure in the range $0 \leq E_{i} \leq 300 \mathrm{eV}$. Note that the former four ions (dominated by $\mathrm{SiCl}_{3}{ }^{+}$at low $E_{i}$, while $\mathrm{Cl}_{2}{ }^{+}$at high $E_{i}$ ) are reactive on $\mathrm{Si}$, while the latter three (dominated by $\mathrm{SiCl}^{+}$) are depositive.

function of ion incident energy in the range $E_{i} \approx 13-570 \mathrm{eV}$, measured with the ionizer off during ICP plasma etching of $\mathrm{Si}$ in $\mathrm{Cl}_{2}$ for four different flow rates of $F_{0}=5-50 \mathrm{sccm}$ under otherwise the same conditions as in Fig. 2. These data are those obtained by summing the respective ion intensities as shown in the preceding Fig. 16, and inset is an enlarged view of the figure in the range $0 \leq E_{i} \leq 300 \mathrm{eV}$. It should be noted here that the former four ions (dominated by $\mathrm{Cl}_{2}{ }^{+}$at low $E_{i}$, while $\mathrm{SiCl}_{3}{ }^{+}$at high $E_{i}$ ) are reactive on $\mathrm{Si}$, while the latter three (dominated by $\mathrm{SiCl}^{+}$) are depositive, as has been demonstrated in our recent MD simulations of $\mathrm{SiCl}_{x}{ }^{+}$ $(x=0-4)$ and $\mathrm{Cl}_{x}{ }^{+}(x=1,2)$ ions normally incident $\left(\theta_{i}=0^{\circ}\right)$ on $\mathrm{Si}(100)$ surfaces with translational energies in the range $E_{i}=20-500 \mathrm{eV} ;{ }^{113}$ in effect, in $\mathrm{SiCl}_{3}{ }^{+}, \mathrm{SiCl}_{4}{ }^{+}, \mathrm{Cl}^{+}$, and $\mathrm{Cl}_{2}{ }^{+}$incidences, the etching occurs in the whole $E_{i}$ range investigated; on the other hand, in $\mathrm{SiCl}^{+}$and $\mathrm{SiCl}_{2}{ }^{+}$incidences, the deposition occurs at low $E_{i}<300$ and $150 \mathrm{eV}$, respectively, while the etching occurs at further increased $E_{i}$; in addition, in the $\mathrm{Si}^{+}$incidence, the deposition occurs in the whole $E_{i}$ range investigated.

The results indicate that as the bias power $P_{\text {rf }}$ or ion energy $E_{i}$ is increased, the total intensity or flux of reactive ions decreases, while that of depositive ones increases; consequently, the change in the predominant ion flux from reactive to depositive occurs at increased $E_{i}$, where the transition point is increased for increased $F_{0}$, being at around $E_{i} \approx 30$, 70,150 , and $500 \mathrm{eV}$ for $F_{0}=5,10,20$, and $50 \mathrm{sccm}$, respectively. Such a change in the predominant ion flux appears to be related to the two modes of surface roughening that occur 
depending on $E_{i}$, as shown and discussed above in Secs. III B and III C, although the transition point concerned does not fully agree with the transition point $E_{p}$ for the rms roughness versus $E_{i}$ curve [Fig. 4(b)] and that for the ER or $Y$ versus $\sqrt{E_{i}}$ curve (Fig. 5).

\section{E. Mechanisms for surface roughening}

Figure 18 shows the etch rate and rms surface roughness of $\mathrm{Si}$ as a function of ion incident energy in the rage $E_{i}=20-500 \mathrm{eV}$, obtained through $\mathrm{ICP} \mathrm{Cl}_{2}$ plasma etching experiments and ASCeM-3D simulations $\left(t_{\mathrm{etch}}=2 \mathrm{~min}\right)$. The experimental data shown are those for $F_{0}=20 \mathrm{sccm}$ in Fig. 4, and the ASCeM-3D results are those calculated with normal ion incidence $\left(\theta_{i}=0^{\circ}\right)$ assuming similar plasma conditions. Note that the ASCeM-3D is our original 3D Monte Carlobased simulation model for plasma-surface interactions and the feature profile evolution during plasma etching of $\mathrm{Si}$ in $\mathrm{Cl}_{2}$ and $\mathrm{Cl}_{2} / \mathrm{O}_{2}$, as has been detailed in our recent paper; ${ }^{45}$ briefly, the model takes into account the behavior (transport and surface reactions) of $\mathrm{Cl}^{+}$ions, $\mathrm{Cl}$ and $\mathrm{O}$ neutrals, and etch products and byproducts of $\mathrm{SiCl}_{x}$ and $\mathrm{SiCl}_{x} \mathrm{O}_{y}$ in microstructures and on feature surfaces therein. The surface chemistry and kinetics included are surface chlorination, chemical etching, ion-enhanced etching, sputtering, surface oxidation, redeposition of etch products desorbed from feature surfaces being etched, and deposition of etch products/byproducts coming from the plasma. The model also takes into account the ion reflection or scattering from feature surfaces on incidence and/or the ion penetration into substrates, along with geometrical shadowing of the feature and surface reemission of neutrals. The simulation domain consists of small cubic cells of atomic size ( $L=2.7 \AA$ ), and the evolving interfaces are represented by removing $\mathrm{Si}$ atoms from and/or allocating them to the cells concerned. Calculations were performed for square substrates $W=50 \mathrm{~nm}$ on a side, with an incoming ion flux and neutral reactant-to-ion flux ratio of $\Gamma_{i}^{0}=1.0 \times 10^{16} \mathrm{~cm}^{-2} \mathrm{~s}^{-1}$ and $\Gamma_{n}^{0} / \Gamma_{i}^{0}=100$, respectively, in the absence of incoming oxygen and etch products/byproducts $\left(\Gamma_{o}{ }^{0}=\Gamma_{p}{ }^{0}=0\right){ }^{45}$ in addition, the ion and neutral (or gas) temperatures were assumed to be $k_{B} T_{i}=0.5 \mathrm{eV}$ and $T_{g}=500 \mathrm{~K}$, respectively, together with the dopant concentration $N_{e}=1.0 \times 10^{18} \mathrm{~cm}^{-3}$ and surface temperature $T_{s}=320 \mathrm{~K}$ of substrates.
A comparison between experiments and simulations indicates that the ASCeM-3D reproduces well the increase in Si etch rate with increasing $E_{i}$ in the whole range investigated, and the increase in rms roughness of etched Si surfaces at low $E_{i}<E_{p} \approx 250 \mathrm{eV}$ (roughening mode); in contrast, it does not reproduce the decrease in rms surface roughness of $\mathrm{Si}$ at higher $E_{i}>E_{p}$ (smoothing mode), where the rms roughness in experiments decreases substantially with increasing $E_{i}$ down to a low level $<0.4 \mathrm{~nm}$, while it continues to increase with $E_{i}$ in simulations. Some difference in magnitude of the rms roughness between experiments and simulations at low $E_{i}<E_{p}$ is ascribed to the known behavior that the larger the scan area, the larger is the roughness; ${ }^{114}$ in practice, the rms roughness in experiments is that measured for the AFM scan area of $1 \times 1 \mu \mathrm{m}^{2}$ (as mentioned earlier with respect to Fig. 3), while the rms roughness in simulations is that acquired for the ASCeM-3D domain of $50 \times 50 \mathrm{~nm}^{2}$.

\section{Smoothing mode}

Also shown in Fig. 18 are the ASCeM-3D results for $\mathrm{Si}$ etching in $\mathrm{Cl}_{2}$ plasmas obtained with the ion reflection switched off in the simulation. The results indicate that in the absence of ion reflection (or assuming all the ions incident on surfaces to penetrate into substrates without reflection), the rms surface roughness is reduced substantially to a low level $<0.4 \mathrm{~nm}$, almost independently of ion energy in the whole $E_{i}$ range investigated, and surprisingly it is in agreement with the experiments at high $E_{i}>400 \mathrm{eV}$. It is noted that the etch rate is slightly increased with the ion reflection off, owing to the increased ion energy deposited on surfaces, which is more significant at increased $E_{i}$. Such a substantial decrease in rms roughness and slight increase in etch rate with the ion reflection off were also observed in the ASCeM-3D simulations taking into account of incoming oxygen and etch products/byproducts (as will be partly shown later). These imply that the ion reflection or scattering from microscopically roughened feature surfaces on incidence plays a crucial role in plasma-induced surface roughening, particularly in the evolution of surface roughness with time during etching; in effect, the reflection of energetic ions from feature surfaces tends to concentrate them onto the
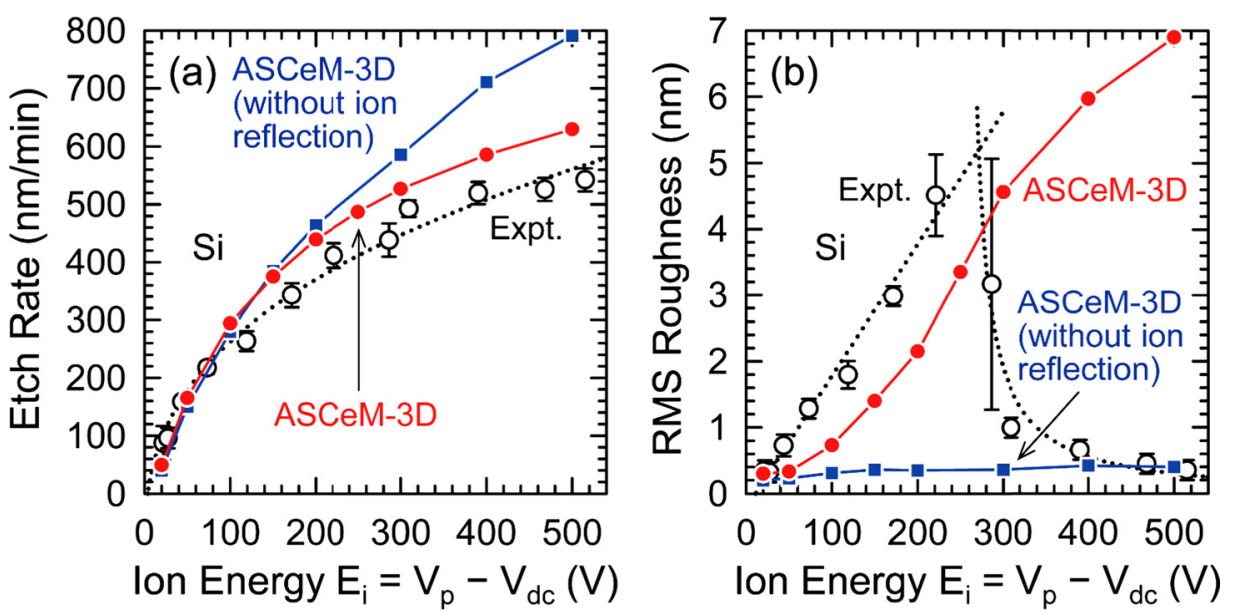

FIG. 18. (a) Etch rate and (b) rms surface roughness of $\mathrm{Si}$ as a function of ion incident energy in the range $E_{i}$ $=20-500 \mathrm{eV}$, obtained through ICP $\mathrm{Cl}_{2}$ plasma etching experiments and ASCeM-3D simulations $\left(t_{\text {etch }}=2 \mathrm{~min}\right)$. Also shown are the ASCeM-3D results obtained with the ion reflection switched off in the simulation. The experimental data shown are those for $F_{0}=20 \mathrm{sccm}$ in Fig. 4, and the ASCeM-3D results are those calculated with the simulation domain of $50 \times 50 \mathrm{~nm}^{2}$ under normal ion incidence $\left(\theta_{i}=0^{\circ}\right)$ assuming similar plasma conditions [see the text for the calculation conditions $\left.\left(\Gamma_{o}^{0}=\Gamma_{p}^{0}=0\right)\right]$. 
bottom (or the valley) of the feature, ${ }^{44}$ thus resulting in an increase in roughness to its vertical as well as lateral extent, where the increase in lateral extent of the roughness is attributed to the uniformity of the incident flux and angle of ions and/or neutral etchants on surfaces at microscale. Thus, at increased $E_{i}>E_{p}$ (smoothing mode) in the present experiments, some plasma environments (or parameters of incoming ions) may be changed to significantly reduce the effects of ion reflection and thus substantially reduce the surface roughness down to the low level of stochastic roughening. A promising change of interest is that in the predominant flux of ionic species from feed gas ions $\mathrm{Cl}_{x}{ }^{+}$to ionized etch products/byproducts $\mathrm{SiCl}_{x}^{+}$at increased $E_{i}$, caused by the increased etch rates, as shown in Figs. 16 and 17; more precisely, the change in the predominant ion flux from reactive ions (dominated by $\mathrm{Cl}_{2}{ }^{+}$at low $E_{i}$, while $\mathrm{SiCl}_{3}{ }^{+}$at high $E_{i}$ ) to depositive ones (dominated by $\mathrm{SiCl}^{+}$) at increased $E_{i}$.

In practice, our recent $\mathrm{MD}$ simulations of $\mathrm{SiCl}_{x}^{+}$ $(x=0-4)$ and $\mathrm{Cl}_{x}{ }^{+}(x=1,2)$ ion incidences on $\mathrm{Si}(100)$ surfaces with translational energies of $E_{i}=20-500 \mathrm{eV}$ have demonstrated that for any $E_{i}$, the reflection probability on surfaces increases with increasing incidence angle in the range $\theta_{i}=0^{\circ}-85^{\circ}$ investigated, where it is significantly lowered for depositive ions $\left(\mathrm{Si}^{+}, \mathrm{SiCl}^{+}, \mathrm{SiCl}_{2}{ }^{+}\right)$as compared with that for reactive ones $\left(\mathrm{SiCl}_{3}{ }^{+}, \mathrm{SiCl}_{4}{ }^{+}, \mathrm{Cl}^{+}, \mathrm{Cl}_{2}{ }^{+}\right) ;{ }^{115}$ particularly, at grazing incidence $\left(\theta_{i} \geq 80^{\circ}\right)$, the reflection probability was estimated to be in the range $0.6-0.95$ for depositive ions (depending on species and $E_{i}$ ), while it was to be approximately unity for reactive ones (almost independently of species and $E_{i}$ ). For example, the reflection probability at $\theta_{i}=80^{\circ}$ was estimated to be about $0.99,0.96$, and 0.83 for $\mathrm{SiCl}_{3}{ }^{+}, \mathrm{Cl}_{2}{ }^{+}$, and $\mathrm{SiCl}^{+}$with $E_{i}=50 \mathrm{eV}$, respectively, and it was to be about $0.99,0.99$, and 0.90 for $\mathrm{SiCl}_{3}{ }^{+}, \mathrm{Cl}_{2}{ }^{+}$, and $\mathrm{SiCl}^{+}$with $E_{i}=500 \mathrm{eV}$, respectively. Moreover, our recent ASCeM-3D simulations have demonstrated that with the reflection of at least $10-20 \%$ of the incident ions switched off (or assuming at least $10-20 \%$ of the ions incident on surfaces to penetrate into substrates without reflection), the rms surface roughness of $\mathrm{Si}$ is reduced substantially to a low level $<0.4 \mathrm{~nm}$, almost independently of $E_{i}$, similarly as in Fig. $18 .{ }^{116}$ These would support our proposed mechanisms as mentioned above, for the phenomena presently observed at increased $E_{i}>E_{p}$ (smoothing mode) in experiments.

\section{Roughening mode}

Figures 19(a) and 19(b) show the etch rate and rms surface roughness of $\mathrm{Si}$ as a function of etching or plasma exposure time in the range $0 \leq t \leq 2 \mathrm{~min}$ for five different ion incident energies of $E_{i}=20-500 \mathrm{eV}$, obtained through ASCeM-3D simulations with the ion reflection switched on and off for $\mathrm{Si}$ etching in $\mathrm{Cl}_{2}$ plasmas. These are the respective typical runs, corresponding to the ASCeM-3D results in the preceding Fig. 18 . For any $E_{i}$, the etch rate exhibits a significant increase immediately after the start of etching and then maintains an almost constant value, in both the presence and absence of the effects of ion reflection; on the other
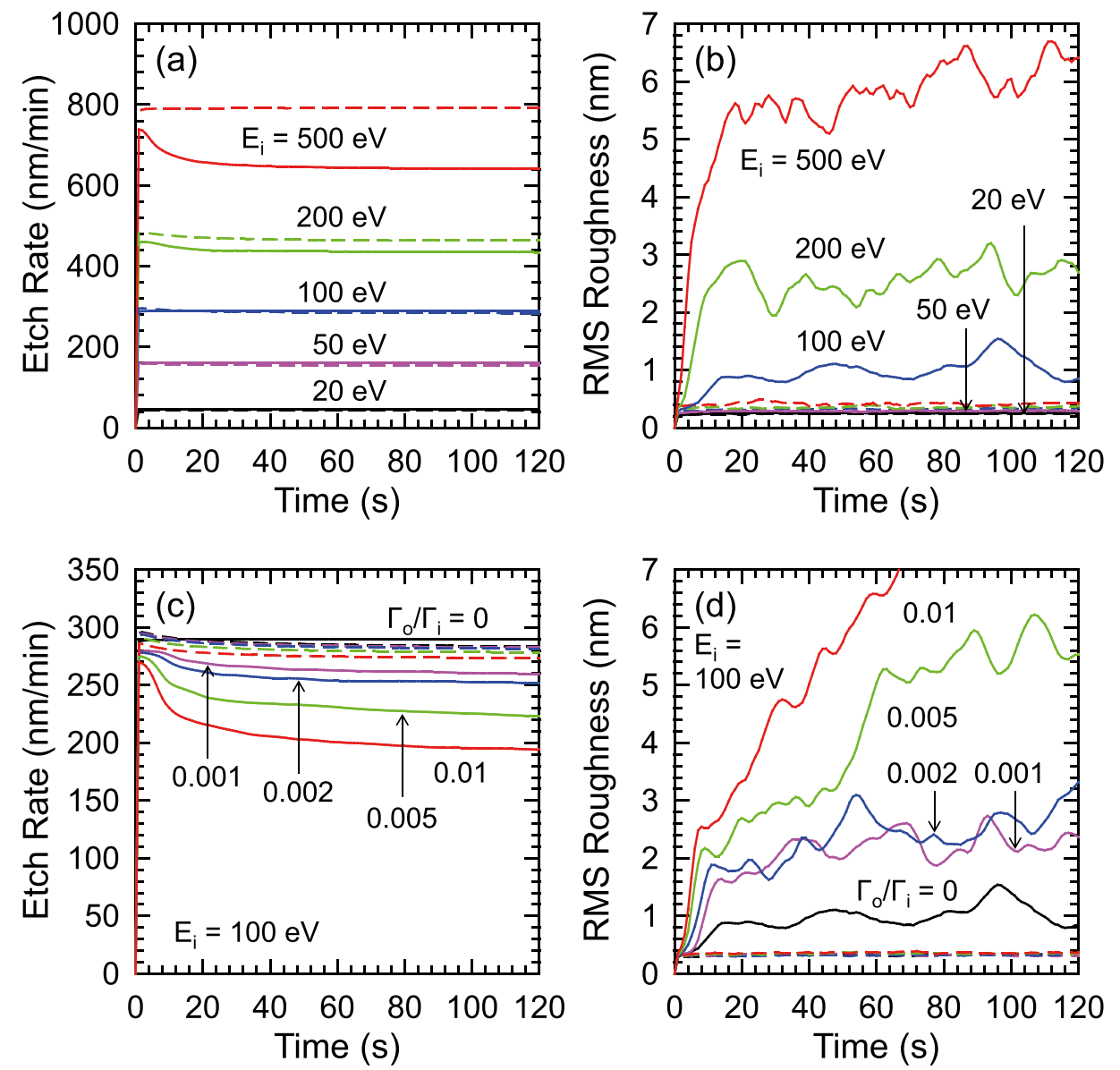

FIG. 19. (a) Etch rate and (b) rms surface roughness of $\mathrm{Si}$ as a function of etching or plasma exposure time in the range $0 \leq t \leq 2 \mathrm{~min}$ for five different ion incident energies of $E_{i}=20-500$ $\mathrm{eV}$, obtained through ASCeM-3D simulations with the ion reflection switched on (solid lines) and off (broken lines) for $\mathrm{Si}$ etching in $\mathrm{Cl}_{2}$ plasmas. These are the respective typical runs, corresponding to the ASCeM-3D results shown in the preceding Fig. 18. Also shown are the (c) etch rate and (d) rms roughness of $\mathrm{Si}$ as a function of time, which are typical runs in the range $0 \leq t \leq 2 \mathrm{~min}$ for $E_{i}=100 \mathrm{eV}$ and five different incoming oxygen-toion flux ratios of $\Gamma_{o}^{0} / \Gamma_{i}^{0}=0-0.01$, obtained through ASCeM-3D simulations with the ion reflection on (solid lines) and off (broken lines) for $\mathrm{Si}$ etching in $\mathrm{Cl}_{2} / \mathrm{O}_{2}$ plasmas under otherwise the same calculation conditions as in Fig. 18. Note that the data for $\Gamma_{o}{ }^{0} / \Gamma_{i}^{0}=0$ in (c) and (d) are the same as those for $E_{i}=100 \mathrm{eV}$ in (a) and (b). 
hand, the rms roughness increases with time for a while $(t<20 \mathrm{~s})$ and then tends to reach quasi-steady state in the presence of ion reflection, while it reaches quasi-steady state immediately after the start of etching, remaining at a low level $<0.4 \mathrm{~nm}$ in the absence of ion reflection. Thus, it follows that the ASCeM-3D does not reproduce the almost linear increase in rms surface roughness of $\mathrm{Si}$ with time during etching, presently observed at low $E_{i}<E_{p} \approx 250 \mathrm{eV}$ (roughening mode) in experiments as shown in Fig. 7, although it reproduces well the increase in $\mathrm{Si}$ etch rate and rms roughness with $E_{i}$ thereat as in Fig. 18. In addition, it may also follow that in a sense, the ASCeM-3D with the ion reflection off reproduces the quasi-steady $\mathrm{rms}$ surface roughness of $\mathrm{Si}$ at a low level, observed at higher $E_{i}>E_{p}$ (smoothing mode) in experiments as shown in Fig. 7, along with the continuous increase in Si etch rate with $E_{i}$ and its substantially decreased rms roughness thereat as in Fig. 18.

An increase in rms surface roughness with time during etching, along with quasi-steady etch rates, has been observed in several plasma etching experiments of $\mathrm{Si}$ in $\mathrm{SF}_{6}$ (Refs. 9, 20, 23, 26) and $\mathrm{CF}_{4} / \mathrm{O}_{2}{ }^{14}$ being attributed to the effects of etch inhibitors coming from the plasma. ${ }^{20,26}$ The corresponding 2D and 3D Monte Carlo simulations of the surface feature evolution reproduced an almost linear increase in rms roughness and correlation length with time, assuming the origins for inhibitors to be materials of the reactor walls, electrodes, and etching masks (hard inhibitors), in addition to less volatile etch products/byproducts and feed gas fragments (soft inhibitors). ${ }^{20,26}$ Similarly, the ASCeM-3D simulations taking into account incoming inhibitor fluxes of oxygen $\left(\Gamma_{o}{ }^{0} \neq 0\right)$ exhibited an increase in rms surface roughness of $\mathrm{Si}$ with increasing oxygen flux added, along with a significant decrease in etch rate, ${ }^{45}$ reproducing roughly an increase in rms roughness with time, as below.

Figures 19(c) and 19(d) show the Si etch rate and rms surface roughness as a function of time in the range $0 \leq t \leq 2 \mathrm{~min}$ for $E_{i}=100 \mathrm{eV}$ and five different oxygen-toion flux ratios of $\Gamma_{o}^{0} / \Gamma_{i}^{0}=0-0.01$, obtained through ASCeM-3D simulations with the ion reflection switched on and off for $\mathrm{Si}$ etching in $\mathrm{Cl}_{2} / \mathrm{O}_{2}$ plasmas under otherwise the same calculation conditions as in Fig. 18. Note that the data for $\Gamma_{o}^{0} / \Gamma_{i}^{0}=0$ in Figs. 19(c) and 19(d) are the same as those for $E_{i}=100 \mathrm{eV}$ in Figs. 19(a) and 19(b). The etch rate behavior with incoming oxygen added is similar to that without oxygen, in both the presence and absence of the effects of ion reflection, where the decrease in $\mathrm{Si}$ etch rate with oxygen added is ascribed to surface oxidation that occurs; ${ }^{45}$ in addition, the slightly increased etch rate with the ion reflection off is more significant for increased $\Gamma_{o}^{0} / \Gamma_{i}^{0}$, because the increased ion energy deposited on surfaces would increase the sputtering of oxides off the surface. On the other hand, the behavior of the rms roughness with incoming oxygen added is different from that without oxygen in the presence ion reflection: the rms surface roughness of $\mathrm{Si}$ tends to increase with time with oxygen added, which is more significant for increased $\Gamma_{o}^{0} / \Gamma_{i}^{0}$. In addition, it is noted that the rms roughness is reduced substantially with the ion reflection switched off, remaining at a low level $<0.4 \mathrm{~nm}$, whether there are incoming oxygen fluxes or not. A small amount of oxygen of the order of $\Gamma_{o}^{0} / \Gamma_{i}^{0} \approx 10^{-3}$ (or $\Gamma_{o}{ }^{0} / \Gamma_{n}{ }^{0} \approx 10^{-5}$ ) concerned here is ascribed to $\mathrm{O}$ impurities sputtered from the dielectric window at the top of the ICP reactor chamber under plasma-on conditions, as mentioned in Sec. IIID 3 with respect to Fig. 15.

The ASCeM-3D simulations taking into account incoming inhibitor fluxes of etch products/byproducts $\left(\Gamma_{p}^{0} \neq 0\right)$ also exhibited an increase in rms surface roughness of $\mathrm{Si}$ with increasing product/byproduct flux added, along with a little decrease in etch rate, ${ }^{45}$ while they did not reproduce an increase in rms roughness with time; in these calculations, the time evolution of the rms surface roughness of Si was similar to that in the absence of incoming etch inhibitors as in Fig. 19(b), or the rms roughness was found to increase for a while after the start of etching and then soon reach quasisteady state. The decrease in $\mathrm{Si}$ etch rate with incoming products/byproducts added is ascribed to deposition of products/byproducts on surfaces being etched. ${ }^{45}$ It should be noted that the two kinds of incoming etch inhibitors concerned here, oxygen and etch products/byproducts, are assumed to play a similar role in surface chemistry and kinetics during etching of $\mathrm{Si}^{45}$ they stick and/or deposit on surfaces being etched, to form passivation layers and inhibit etching thereon; in particular, on microscopically roughened feature surfaces, they are expected to stick and/or deposit preferentially on the top (or the hill) of roughened surface features, owing to the effects of neutral shadowing of the feature, and so tend to locally form passivation layers (or micromasks) and inhibit etching thereon, which leads to an increase in roughness with time during etching. The surface inhibitor or passivation layers thus formed (by surface oxidation and deposition of products/byproducts) are being removed by sputtering through energetic ion bombardment thereon. It should be further noted that in these situations, the sputter yields for energetic ions incident are assumed to be significantly (typically one order of magnitude) smaller on oxidized surfaces of $\mathrm{SiO}_{x}$ than on deposited ones of $\mathrm{SiCl}_{x}$ and $\mathrm{SiCl}_{x} \mathrm{O}_{y},{ }^{45}$ which in turn would lead to the difference in the evolution of roughness between the two kinds of inhibitor species concerned: an increase in roughness as well as a decrease in etch rate with incoming inhibitor fluxes added is more significant for oxygen than for products/byproducts. Hence, these imply that the almost linear increase in rms roughness of $\mathrm{Si}$ with time during etching, presently observed at low $E_{i}<E_{p}$ (roughening mode) in experiments, is caused presumably by a small amount of impurity oxygen contained.

\section{Overview of mechanisms}

Figure 20 shows a schematic of the mechanisms responsible for surface roughening during plasma etching of Si discussed and proposed above: (a) the low-level surface roughening occurs during etching, owing to the stochastic roughening that originates intrinsically from the temporal as well as spatial uniformity of the incident flux and angle of ions and/or neutral etchants on surfaces at microscale. ${ }^{13-16,21,24,45}$ (b) The ion reflection or scattering from microscopically roughened feature surfaces on incidence is 


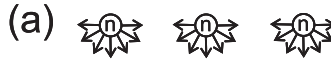

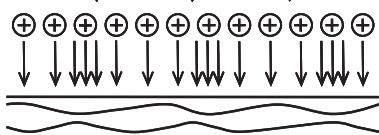

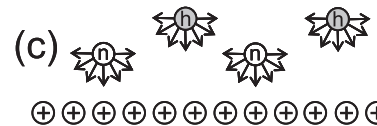

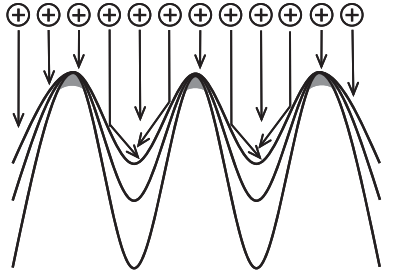

$\oplus$ lons
(10) Neutral etchants

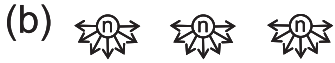
$\oplus \oplus \oplus \oplus \oplus \oplus \oplus \oplus \oplus \oplus \oplus \oplus$

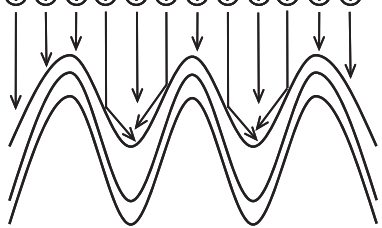

(d)

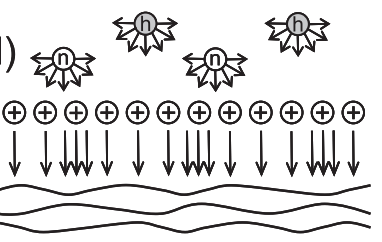

(b) Inhibitors
FIG. 20. Schematic of the mechanisms responsible for surface roughening during plasma etching of Si discussed and proposed: (a) the low-level stochastic roughening occurs during etching, owing intrinsically to the temporal as well as spatial uniformity of the incident flux and angle of ions and/or neutral etchants on surfaces at microscale. (b) The ion reflection or scattering from microscopically roughened feature surfaces on incidence is responsible for the evolution of surface roughness with time during etching, which tends to be limited by the shadowing effects of the feature for neutral etchants. (c) The continuous increase in the roughness with time (roughening mode) is attributed to the effects of a small amount of incoming etch inhibitors, such as oxygen, which tend to locally form surface passivation layers (or micromasks) and inhibit etching thereon, owing to the shadowing for neutral inhibitors. (d) In situations, where the predominant ion fluxes are those with reduced reflection probabilities on surfaces, the roughness tends to remain at a low level of stochastic roughening (smoothing or nonroughening mode), whether there are incoming etch inhibitor fluxes or not. Note that in these illustrations [particularly in (a) and (d)], the cross section is not in scale and the vertical scale is much less than the lateral one, and that in (b) and (c), the increase in the lateral extent of the roughness with time is not illustrated for simplicity.

responsible for the evolution of surface roughness with time during etching, because it tends to concentrate incident ions onto the bottom (or the valley) of the feature, ${ }^{44}$ thus resulting in an increase in roughness to its vertical as well as lateral extent. However, the evolution of the roughness would be limited by the shadowing effects of the feature for neutral etchants, where the deeper the feature, the lower is the etchant flux onto the bottom of the feature. (c) The continuous increase in the roughness with time (roughening mode) is attributed to the effects of a small amount of incoming etch inhibitors, such as oxygen and etch products/byproducts: they stick and/or deposit preferentially on the top (or the hill) of the feature, owing to the shadowing for neutral inhibitors, and so tend to locally form surface passivation layers (or micromasks) and inhibit etching thereon. Such effects of inhibitors are expected to be highly significant for oxygen, because the sputter yields for energetic ions incident are typically one order of magnitude smaller on oxidized than on deposited surfaces. Note that in these illustrations [particularly, in Figs. 20(a) and 20(d)], the cross section is not in scale and the vertical scale is much less than the lateral one, and that in Figs. 20(b) and 20(c), the increase in the lateral extent of the roughness with time is not illustrated for simplicity.

On the other hand, (d) in situations where the predominant ion fluxes are those with reduced reflection probabilities on surfaces, the roughness tends to remain at a low level of stochastic roughening (smoothing or non-roughening mode), whether there are incoming etch inhibitor fluxes or not. In practice, the transition from roughening to smoothing modes was found to occur at increased $E_{i}$ in the present experiments of $\mathrm{Si}$ etching in $\mathrm{Cl}_{2}$ plasmas, corresponding to changes in the predominant ion flux from reactive ions (dominated by $\mathrm{Cl}_{2}{ }^{+}$ at low $E_{i}$, while $\mathrm{SiCl}_{3}{ }^{+}$at high $E_{i}$ ) to depositive ones (dominated by $\mathrm{SiCl}^{+}$) thereat.

\section{CONCLUSIONS}

Atomic- or nanometer-scale surface roughening has been investigated during $\mathrm{Si}$ etching in $\mathrm{ICP} \mathrm{Cl}_{2}$ plasmas, with emphasis being placed on a better understanding of plasmasurface interactions that are responsible for. Experiments were performed at a constant pressure $\left(P_{0}=20 \mathrm{mT}\right.$ Torr $)$ as a function of $\mathrm{rf}$ bias power or ion incident energy in the range $E_{i} \approx 20-500 \mathrm{eV}$, by varying the feed gas flow rate $\left(F_{0}=5-50 \mathrm{sccm}\right)$, wafer stage temperature $\left(T_{s}=20-200^{\circ} \mathrm{C}\right)$, and etching or plasma exposure time $\left(t_{\text {etch }}=1-20 \mathrm{~min}\right)$, including several plasma and surface diagnostics. The experiments revealed two modes of plasma-induced surface roughening which occur depending on $E_{i}$ : roughening and smoothing (or non-roughening) modes. In the roughening mode at low $E_{i}<E_{p} \approx 200-300 \mathrm{eV}$ (depending slightly on $F_{0}$ ), the rms roughness of etched surfaces as well as the etch rate increases with increasing $E_{i}$, where the etch rate or yield versus $\sqrt{E_{i}}$ curve exhibits a relatively large slope or proportional constant; moreover, the rms roughness increases almost linearly with time during etching $(t<20 \mathrm{~min})$, where the higher the $E_{i}$, the larger is the increase in roughness. The roughness evolution with time does not follow that of the etch rate at low $E_{i}<E_{p}$, because the etch rate reaches quasisteady state soon after the start of etching in the whole $E_{i}$ range investigated. On the other hand, in the soothing mode at higher $E_{i}>E_{p}$, the rms surface roughness decreases substantially with increasing $E_{i}$ down to a low level $<0.4 \mathrm{~nm}$ (initially $\sim 0.15 \mathrm{~nm}$ ), while the etch rate continues to increase with $E_{i}$, where the etch rate or yield versus $\sqrt{E_{i}}$ curve exhibits a decreased proportional constant; moreover, the rms roughness tends to reach quasi-steady state after some increase at the initial stage of etching $(t<1 \mathrm{~min})$, where the higher the $E_{i}$, the smaller is the roughness at steady state.

Correspondingly, the power spectral density analysis exhibited two different behaviors of the roughness evolution: the spectral distributions in the roughening mode consist of a frequency independent regime at low spatial frequencies and a high frequency self-affine regime, where the plateau value and the correlation length increase with time during etching, with the fractal-linked exponent remaining almost unchanged at $\eta \approx 3$; on the other hand, the spectral distributions in the smoothing mode consist of two self-affine regimes at low and high frequencies, where the distributions remain almost unchanged over time $(t>1 \mathrm{~min})$ during etching with the fractal exponents of $\eta \approx 1$ and 3 .

Such changes in surface roughening from the roughening to smoothing modes with increasing $E_{i}$ were found to 
correspond approximately to changes in the predominant ion flux from feed gas ions $\mathrm{Cl}_{x}{ }^{+}$to ionized etch products $\mathrm{SiCl}_{x}{ }^{+}$ caused by increased etch rates at increased $E_{i}$, in view of the results of plasma diagnostics, such as OES, FTIR, and QMS to characterize the plasma during etching; more precisely, the changes in the predominant ion flux from reactive ions (dominated by $\mathrm{Cl}_{2}{ }^{+}$at low $E_{i}$, while $\mathrm{SiCl}_{3}{ }^{+}$at high $E_{i}$ ) to depositive ones (dominated by $\mathrm{SiCl}^{+}$) at increased $E_{i}$.

The low-level surface roughening during plasma etching, usually giving self-affine fractal surfaces, is generally ascribed to the stochastic roughening that originates intrinsically from the temporal as well as spatial uniformity of the incident flux and angle of ions and/or neutral etchants on surfaces at microscale. ${ }^{13-16,21,24,45}$ Possible mechanisms for the evolution of surface roughness during etching were further discussed with the help of Monte Carlo-based ASCeM3D simulations of the surface feature evolution and classical MD simulations of etch fundamentals for Si etching in $\mathrm{Cl}_{2}$ plasmas. A comparison of the etch rate and rms surface roughness between experiments and ASCeM-3D simulations indicated that the ASCeM-3D reproduces well the increase in etch rate and rms roughness with increasing $E_{i}$ at low $E_{i}<E_{p}$ (roughening mode) in experiments, while it does not reproduce the almost linear increase in rms roughness with time during etching thereat; on the other hand, the ASCeM3D does not reproduce the substantial decrease in rms roughness with increasing $E_{i}$ at higher $E_{i}>E_{p}$ (smoothing mode) in experiments, although it reproduces well the continuous increase in etch rate with $E_{i}$ thereat, and in a sense, it reproduces the quasi-steady rms roughness soon after the start of etching thereat.

In these situations, the ASCeM-3D with the ion reflection switched off in the simulation exhibited a substantial decrease in rms surface roughness of $\mathrm{Si}$ down to a low level $<0.4 \mathrm{~nm}$ (almost independently of $E_{i}$ ) without a significant change in etch rate, which is in agreement with experiments at high $E_{i}>E_{p}$. These imply that the ion reflection or scattering from microscopically roughened feature surfaces on incidence plays a crucial role in plasma-induced surface roughening, particularly in the evolution of surface roughness with time during etching; correspondingly, the change in the predominant ion flux from reactive to depositive ions at increased $E_{i}$ is probably responsible for the change in surface roughening from the roughening to smoothing modes with increasing $E_{i}$, because the ion reflection probability on surfaces is significantly lowered for depositive ions as compared with that for reactive ones, as indicated by the MD simulations concerned. Moreover, the ASCeM-3D simulations taking into account incoming etch inhibitor fluxes of oxygen exhibited a continuous increase in rms surface roughness of $\mathrm{Si}$ with time, where the rms roughness is increased and the etch rate is decreased with increasing oxygen flux added; the decrease in etch rate is attributed to the surface oxidation that occurs, while the increase in roughness is to local surface oxidation or the local formation of oxide micromasks on $\mathrm{Si}$ surfaces during etching. These imply that a small amount of impurity oxygen of the order of $\Gamma_{o}^{0}$ \% $\Gamma_{i}^{0} \approx 10^{-3}$ (or $\Gamma_{o}{ }^{0} / \Gamma_{n}{ }^{0} \approx 10^{-5}$ ) is presumably responsible for the almost linear increase in rms roughness with time during etching in experiments at low $E_{i}<E_{p}$; in practice, the origin of oxygen concerned is attributed to $\mathrm{O}$ impurities sputtered from the dielectric window of the ICP reactor chamber under plasma-on conditions, and the effects of oxygen may be reduced at increased $E_{i}>E_{p}$, owing to the increased removal yields for $\mathrm{O}$ adsorbed through energetic ion bombardment as well as to the reduced effects of ion reflection thereat.

Further studies are now in progress for a more comprehensive understanding of the mechanisms responsible for the formation and evolution of surface roughness during plasma etching; in particular, the transient behavior of plasma environments after the start of etching is of interest in the smoothing mode at high $E_{i}>E_{p}$, because it may be responsible for the roughness evolution at the initial stage of etching and thus the following quasi-steady rms roughness that is decreased down to a low level at increased $E_{i} \gg E_{p}$. In practice, the plasma environments at high $E_{i}>E_{p}$ are considered to change from feed gas-rich to etch product/byproduct-rich regimes in a while after the start of etching, which will be more rapid at increased $E_{i}$ owing to increased etch rates. Moreover, a more comprehensive ASCeM-3D model is required to simulate the incidence of ionized etch products/ byproducts $\mathrm{SiCl}_{x}^{+}$as well as feed gas ions $\mathrm{Cl}_{x}^{+}$on substrate surfaces, because the present ASCeM-3D cannot simulate the $\mathrm{SiCl}_{x}^{+}$ion incidence, which is contradictory to the basic assumption of the ASCeM that its cell is rigid and contains one $\mathrm{Si}$ atom therein. ${ }^{43-45}$

\section{ACKNOWLEDGMENTS}

This work was supported by a Grant-in-Aid for Scientific Research on Innovative Areas (Frontier science of interactions between plasmas and nano-interfaces) from the Ministry of Education, Culture, Sports, Science and Technology, Japan. The authors would like to thank H. Matsumoto for assistance with experiments and T. Hatsuse for assistance with ASCeM-3D simulations.

${ }^{1}$ H. Abe, M. Yoneda, and N. Fujiwara, Jpn. J. Appl. Phys. 47, 1435 (2008).

${ }^{2}$ V. M. Donnelly and A. Kornblit, J. Vac. Sci. Technol. A 31, 050825 (2013).

${ }^{3}$ B. Wu, A. Kumar, and S. Pamarthy, J. Appl. Phys. 108, 051101 (2010).

${ }^{4}$ International Technology Roadmap for Semiconductors (ITRS) 2013 edition, http://www.itrs.net.

${ }^{5}$ K. J. Kanarik, G. Kamarthy, and R. A. Gottscho, Solid State Technol. 55(3), 15 (2012).

${ }^{6}$ E. Pargon, M. Darnon, O. Joubert, T. Chevolleau, L. Vallier, L. Mollard, and T. Lill, J. Vac. Sci. Technol. B 23, 1913 (2005).

${ }^{7}$ P. Pan and L. Liu, J. Vac. Sci. Technol. B 15, 1752 (1997).

${ }^{8}$ D. J. Thomas, P. Southworth, M. C. Flowers, and R. Greef, J. Vac. Sci. Technol. B 7, 1325 (1989).

${ }^{9}$ R. Pétri, P. Brault, O. Vatel, D. Henry, E. André, P. Dumas, and F. Salvan, J. Appl. Phys. 75, 7498 (1994).

${ }^{10}$ H. Feil, J. Dieleman, and B. J. Garrison, J. Appl. Phys. 74, 1303 (1993).

${ }^{11}$ K.-T. Sung and S. W. Pang, Jpn. J. Appl. Phys. Part 1 33, 7112 (1994).

${ }^{12}$ M. J. M. Vugts, M. F. A. Eurlings, L. J. F. Hermans, and H. C. W. Biejerinck, J. Vac. Sci. Technol. A 14, 2780 (1996).

${ }^{13}$ P. Brault, P. Dumas, and F. Salvan, J. Phys. Condens. Matter 10, L27 (1998).

${ }^{14}$ Y.-P. Zhao, J. T. Drotar, G.-C. Wang, and T.-M. Lu, Phys. Rev. Lett. 82, 4882 (1999).

${ }^{15}$ J, T. Drotar, Y.-P. Zhao, T.-M. Lu, and G.-C. Wang, Phys. Rev. B 61, 3012 (2000). 
${ }^{16}$ J. T. Drotar, Y.-P. Zhao, T.-M. Lu, and G.-C. Wang, Phys. Rev. B 62, 2118 (2000).

${ }^{17}$ E. Gogolides, C. Boukouras, G. Kokkoris, O. Brani, A. Tserepi, and V. Constantoudis, Microelectron. Eng. 73-74, 312 (2004).

${ }^{18}$ A. A. E. Stevens and H. C. W. Beijerinck, J. Vac. Sci. Technol. A 23, 126 (2005).

${ }^{19}$ E. Zakka, V. Constantoudis, and E. Gogolides, IEEE Trans. Plasma Sci. 35, 1359 (2007).

${ }^{20} \mathrm{G}$. Kokkoris, V. Constantoudis, P. Angelikopoulos, G. Boulousis, and E. Gogolides, Phys. Rev. B 76, 193405 (2007).

${ }^{21}$ Y. Yin and H. H. Sawin, J. Vac. Sci. Technol. A 26, 151 (2008).

${ }^{22}$ W. S. Hwang, B.-J. Cho, D. S. H. Chan, S. W. Lee, and W. J. Yoo, J. Electrochem. Soc. 155, H6 (2008).

${ }^{23}$ M. Martina and G. Cunge, J. Vac. Sci. Technol. B 26, 1281 (2008).

${ }^{24}$ W. Guo and H. H. Sawin, J. Phys. D 42, 194014 (2009).

${ }^{25}$ J. J. Végh and D. B. Graves, Plasma Sources Sci. Technol. 19, 045005 (2010).

${ }^{26}$ E. Gogolides, V. Constantoudis, G. Kokkoris, D. Kontziampasis, K. Tsougeni, G. Boulousis, M. Vlachopoulou, and A. Tserepi, J. Phys. D 44, 174021 (2011).

${ }^{27}$ Y. Yin and H. H. Sawin, J. Vac. Sci. Technol. A 25, 802 (2007).

${ }^{28}$ W. Guo and H. H. Sawin, J. Vac. Sci. Technol. A 28, 259 (2010).

${ }^{29}$ X. Hua, S. Engelmann, G. S. Oehrlein, P. Jiang, P. Lazzeri, E. Iacob, and M. Anderle, J. Vac. Sci. Technol. B 24, 1850 (2006).

${ }^{30}$ A. R. Pal, R. L. Bruce, F. Weilnboeck, S. Engelmann, T. Lin, M.-S. Kuo, R. Phaneuf, and G. S. Oehrlein, J. Appl. Phys. 105, 013311 (2009).

${ }^{31}$ G. S. Oehrlein, R. J. Phaneuf, and D. B. Graves, J. Vac. Sci. Technol. B 29, 010801 (2011).

${ }^{32}$ R. L. Bruce, F. Weilnboeck, T. Lin, R. J. Phaneuf, G. S. Oehrlein, B. K. Long, C. G. Willson, J. J. Vegh, D. Nest, and D. B. Graves, J. Appl. Phys. 107, 084310 (2010).

${ }^{33}$ T.-C. Lin, R. L. Bruce, G. S. Oehrlein, R. J. Phaneuf, and H.-C. Kan, Appl. Phys. Lett. 100, 233113 (2012).

${ }^{34}$ Y. Yin, S. Rasgon, and H. H. Sawin, J. Vac. Sci. Technol. B 24, 2360 (2006).

${ }^{35}$ J. Thiault, J. Foucher, J. H. Tortai, O. Joubert, S. Ladis, and S. Pauliac, J. Vac. Sci. Technol. B 23, 3075 (2005).

${ }^{36}$ E. Gogolides, V. Constantoudis, G. P. Patsis, and A. Tserepi, Microelectron. Eng. 83, 1067 (2006).

${ }^{37}$ K. Patel, T.-J. King Liu, and C. J. Spanos, IEEE Trans. Electron Devices 56, 3055 (2009)

${ }^{38}$ X. Sun and T.-J. King Liu, IEEE Trans. Semicond. Manuf. 23, 311 (2010).

${ }^{39}$ E. Altamirano-Sánchez, V. Paraschiv, M. Demand, and W. Boullart, Microelectron. Eng. 88, 2871 (2011).

${ }^{40}$ K. Eriguchi, Y. Takao, and K. Ono, J. Vac. Sci. Technol. A 29, 041303 (2011).

${ }^{41}$ K. S. Chen, A. Ayon, and S. M. Spearing, J. Am. Ceram. Soc. 83, 1476 (2000).

${ }^{42}$ N. Tayebi and A. A. Polycarpou, J. Appl. Phys. 98, 073528 (2005).

${ }^{43}$ H. Tsuda, H. Miyata, Y. Takao, K. Eriguchi, and K. Ono, Jpn. J. Appl. Phys. 50, 08 JE06 (2011).

${ }^{44}$ H. Tsuda, Y. Takao, K. Eriguchi, and K. Ono, Jpn. J. Appl. Phys. 51, 08HC01 (2012).

${ }^{45}$ H. Tsuda, N. Nakazaki, Y. Takao, K. Eriguchi, and K. Ono, J. Vac. Sci. Technol. B 32, 031212 (2014).

${ }^{46}$ K. Ono, M. Tuda, K. Nishikawa, T. Oomori, and K. Namba, Jpn. J. Appl. Phys. Part 1 33, 4424 (1994).

${ }^{47}$ K. Nishikawa, T. Oomori, and K. Ono, J. Vac. Sci. Technol. B 17, 127 (1999).

${ }^{48}$ M. A. Lieberman and A. J. Lichtenberg, Principles of Plasma Discharges and Materials Processing, 2nd ed. (Wiley, Hoboken, NJ, 2005), Chap. 6, p. 172.

${ }^{49}$ K. Tsujimoto, T. Kumihashi, and S. Tachi, Appl. Phys. Lett. 63, 1915 (1993).

${ }^{50}$ C. Steinbrüchel, Appl. Phys. Lett. 55, 1960 (1989).

${ }^{51}$ M. Balooch, M. Moalem, W.-E. Wang, and A. V. Hamza, J. Vac. Sci. Technol. A 14, 229 (1996).

${ }^{52}$ J. P. Chang and H. H. Sawin, J. Vac. Sci. Technol. B 19, 1319 (2001).

${ }^{53}$ in ULSI Technology, edited by C. Y. Chang and S. M. Sze (McGraw-Hill, New York, 1996), p. 248 and p. 705.

${ }^{54}$ L. Sha and J. P. Chang, J. Vac. Sci. Technol. A 22, 88 (2004).

${ }^{55}$ S. A. Vitale, H. Chae, and H. H. Sawin, J. Vac. Sci. Technol. A 19, 2197 (2001).

${ }^{56}$ W. Jin, S. A. Vitale, and H. H. Sawin, J. Vac. Sci. Technol. A 20, 2106 (2002).
${ }^{57}$ N. Nakazaki, H. Tsuda, Y. Takao, K. Eriguchi, and K. Ono, "In situ wafer surface temperature measurement during plasma etching of Si using infrared refection-absorption spectroscopy," Appl. Phys. Express (unpublished).

${ }^{58}$ A. Duparré, J. Ferre-Borrull, S. Gliech, G. Notni, J. Steinert, and J. M. Bennett, Appl. Opt. 41, 154 (2002).

${ }^{59}$ M. Senthilkumar, N. K. Sahoo, S. Thakur, and R. B. Tokas, Appl. Surf. Sci. 252, 1608 (2005).

${ }^{60}$ A. E. Lita and J. E. Sanchez, Jr., J. Appl. Phys. 85, 876 (1999).

${ }^{61}$ D. B. Fenner, J. Appl. Phys. 95, 5408 (2004).

${ }^{62}$ D. Raoufi, Physica B 405, 451 (2010).

${ }^{63}$ M. Nie, T. Mete, and K. Ellmer, J. Appl. Phys. 115, 154905 (2014).

${ }^{64}$ T. Tsuchizawa, C. Takahashi, and S. Matsuo, Jpn. J. Appl. Phys. Part 1 33, 6019 (1994).

${ }^{65}$ V. M. Donnelly, J. Vac. Sci. Technol. A 14, 1076 (1996).

${ }^{66}$ K. H. A. Bogart and V. M. Donnelly, J. Appl. Phys. 86, 1822 (1999).

${ }^{67}$ J. Y. Choe, N. C. M. Fuller, V. M. Donnelly, and I. P. Hermana, J. Vac. Sci. Technol. A 18, 2669 (2000).

${ }^{68}$ R. W. B. Pearse and A. G. Gaydon, The Identification of Molecular Spectra, 3rd ed. (Chapman \& Hall, London, 1963).

${ }^{69}$ K. P. Huber and G. Hertzberg, Molecular Spectra and Molecular Structure: IV. Constants of Diatomic Molecules (Van Nostrand Reinhold, New York, 1979).

${ }^{70} \mathrm{H}$. Bredohl, Ph. Demoulin, Y. Houbrechts, and F. Mélen, J. Phys. B 14, 1771 (1981)

${ }^{71}$ B. P. Ruzsicska, A. Jordan, I. Safarik, O. P. Strausz, and T. N. Bell, Chem. Phys. Lett. 113, 67 (1985).

${ }^{72}$ N. Washida, Y. Matsumi, T. Hayashi, T. Ibuki, A. Hiraya, and K. Shobatake, J. Chem. Phys. 83, 2769 (1985).

${ }^{73}$ D. Sameith, J. P. Mönch, H.-J. Tiller, and K. Schade, Chem. Phys. Lett. 128, 483 (1986).

${ }^{74}$ M. Suzuki, N. Washida, and G. Inoue, Chem. Phys. Lett. 131, 24 (1986).

${ }^{75}$ M. D. Rowe, J. Chem. Soc., Faraday Trans. 2 84, 191 (1988).

${ }^{76}$ P. Ho and W. G. Breiland, Appl. Phys. Lett. 44, 51 (1984).

${ }^{77}$ A. R. Striganov and N. S. Sventitskii, Tables of Spectral Lines of Neutral and Ionized Atoms (IFI/Plenum, New York, 1968).

${ }^{78}$ S. D. Peyerimhoff and R. J. Buenker, Chem. Phys. 57, 279 (1981).

${ }^{79}$ J. C. Tellinghuisen and D. K. Chakraborty, Chem. Phys. Lett. 134, 565 (1987).

${ }^{80}$ P. C. Tellinghuisen, B. Guo, D. K. Chakraborty, and J. Tellinghuisen, J. Mol. Spectrosc. 128, 268 (1988).

${ }^{81}$ F. P. Huberman, J. Mol. Spectrosc. 20, 29 (1966).

${ }^{82}$ R. P. Tuckett and S. D. Peyerimhoff, Chem. Phys. 83, 203 (1984).

${ }^{83}$ J. C. Choi and J. L. Hardwick, J. Mol. Spectrosc. 137, 138 (1989).

${ }^{84}$ I. P. Herman, Optical Diagnostics for Thin Film Processing (Academic, New York, 1996), Chap. 6, p. 175.

${ }^{85} \mathrm{~K}$. Nakamoto, Infrared and Raman Spectra of Inorganic and Coordination Compounds: Part A: Theory and Applications in Inorganic Chemistry, 5th ed. (Wiley, New York, 1997), Sec. II, p. 160 and p. 193.

${ }^{86}$ V. M. Donnelly, J. Appl. Phys. 79, 9353 (1996).

${ }^{87}$ G. Cunge, N. Sadeghi, and R. Ramos, J. Appl. Phys. 102, 093305 (2007).

${ }^{88}$ S. Tinck, W. Boullart, and A. Bogaerts, Plasma Sources Sci. Technol. 20, 045012 (2011).

${ }^{89}$ G. Cunge, R. L. Inglebert, O. Joubert, L. Vallier, and N. Sadeghi, J. Vac. Sci. Technol. B 20, 2137 (2002).

${ }^{90}$ G. Cunge, M. Kogelschatz, and N. Sadeghi, J. Appl. Phys. 96, 4578 (2004).

${ }^{91}$ G. Cunge, D. Vempaire, R. Ramos, M. Touzeau, O. Joubert, P. Bodard, and N. Sadeghi, Plasma Sources Sci. Technol. 19, 034017 (2010).

${ }^{92}$ C. Lee, D. B. Graves, and M. A. Lieberman, Plasma Chem. Plasma Process. 16, 99 (1996).

${ }^{93}$ M. W. Kiehlbauch and D. B. Graves, J. Vac. Sci. Technol. A 21, 116 (2003).

${ }^{94}$ A. Agarwal and M. J. Kushner, J. Vac. Sci. Technol. A 26, 498 (2008).

${ }^{95}$ J. M. Campbell, D. Klapstein, M. Dulick, and P. F. Bernath, Astrophys. J. Suppl. Ser. 101, 237 (1995).

${ }^{96}$ H. Singh, J. W. Coburn, and D. B. Graves, J. Vac. Sci. Technol. A 17, 2447 (1999);18, 299 (2000);19, 718 (2001).

${ }^{97}$ A. W. Kolfschoten, R. A. Haring, A. Haring, and A. E. de Vries, J. Appl. Phys. 55, 3813 (1984).

${ }^{98}$ D. J. Oostra, A. Haring, R. P. van Ingen, and A. E. de Vries, J. Appl. Phys. 64, 315 (1988).

${ }^{99}$ H. F. Winters and J. W. Coburn, Surf. Sci. Rep. 14, 162 (1992).

${ }^{100}$ J. W. Coburn, J. Vac. Sci. Technol. B 12, 1384 (1994).

${ }^{101}$ R. Basner, M. Gutkin, J. Mahoney, V. Tarnovsky, H. Deutsch, and K. Becker, J. Chem. Phys. 123, 054313 (2005). 
${ }^{102}$ K. Becker, J. Mahoney, M. Gutkin, V. Tarnovsky, and R. Basner, Jpn. J. Appl. Phys. Part 1 45, 8188 (2006).

${ }^{103}$ S. J. King and S. D. Price, J. Chem. Phys. 134, 074311 (2011).

${ }^{104}$ J. Mahoney, V. Tarnovsky, and K. H. Becker, Eur. Phys. J. D 46, 289 (2008).

${ }^{105}$ M. Gutkin, J. M. Mahoney, V. Tarnovsky, H. Deutsch, and K. Becker, Int. J. Mass Spectrom. 280, 101 (2009).

${ }^{106}$ P. Calandra, C. S. S. O'Connor, and S. D. Price, J. Chem. Phys. 112, 10821 (2000).

${ }^{107}$ S. Pal, B. Bhatt, and J. Kumar, Int. J. Mass Spectrom. 229, 151 (2003).

${ }^{108}$ P. Mozejko, G. Kasperski, Cz. Szmytkowski, A. Zecca, G. P. Karwasz, L. Del Longo, and R. S. Brusa, Eur. Phys. J. D 6, 481 (1999).

${ }^{109}$ H. N. Kothari, S. H. Pandya, and K. N. Joshipura, J. Phys. B 44, 125202 (2011).

${ }^{110}$ F. A. Stevie and M. J. Vasile, J. Chem. Phys. 74, 5106 (1981).
${ }^{111}$ M. V. Krepa and D. S. Belić, J. Phys. B 11, 3719 (1978).

${ }^{112}$ L. G. Christophorou and J. K. Olthoff, J. Phys. Chem. Ref. Data 28, 131 (1999).

${ }^{113}$ N. Nakazaki, Y. Takao, K. Eriguchi, and K. Ono, Jpn. J. Appl. Phys. 53, 056201 (2014).

${ }^{114}$ K. Boussu, B. Van der Bruggen, A. Volodin, J. Snauwaert, C. Van Haesendonck, and C. Vandecasttele, J. Colloids Interface Sci. 286, 632 (2005).

${ }^{115}$ N. Nakazaki, Y. Takao, K. Eriguchi, and K. Ono, "Molecular dynamics simulations of oblique incidence of silicon chloride ions during Si etching in Cl-based plasmas,” Jpn. J. Appl. Phys. (unpublished).

${ }^{116}$ T. Hatsuse, N. Nakazaki, H. Tsuda, Y. Takao, K. Eriguchi, and K. Ono, "Origin of plasma-induced surface roughening and ripple formation on silicon: A Monte Carlo study,” Jpn. J. Appl. Phys. (unpublished). 